\title{
Protección e importaciones de madera en España (1880-1935)
}

\section{Protection and timber imports in Spain (1880-1935)}

\author{
IÑAKI IRIARTE GOÑI \\ MARÍA ISABEL AYUDA BOSQUE \\ Universidad de Zaragoza
}

RESUMEN

Este trabajo analiza las importaciones españolas de madera no manufacturada y de madera manufacturada entre 1880 y 1935. Para ello, comienza poniendo ambas series en relación con el consumo interior y comprueba el mantenimiento a un nivel elevado de las primeras y la reducción de las segundas.

Después, el trabajo trata de medir la responsabilidad que tuvieron los aranceles en ese diferente comportamiento, calculando la protección aplicada a cada uno de esos grupos y utilizando dos modelos econométricos que

miden la elasticidad de las importaciones respecto a las variaciones del arancel en uno y otro caso. La principal conclusión es que las variaciones en la protección tuvieron un efecto muy moderado en el comportamiento de las importaciones. Ello obliga a explicar el diferente comportamiento de uno y otro tipo de madera por su mayor o menor competitividad respecto a las extranjeras, lo cual se relaciona, a su vez, con las dotaciones de madera del país y con los problemas de integración del mercado maderero nacional.

PALABRAS CLAVE: Madera, Comercio exterior, Sector forestal, España contemporánea Códigos JEL: C22, N53, N54, N73, N74

\section{ABSTRACT}

This work analyses Spanish imports of rough and manufactured timber between 1880 and 1935. We start by measuring the percentage of both over Spanish wood consumption, showing the continuity of the former and the fall of the latter. Then an annual series of tariffs applicable to these products is built in order to estimate, through two different econometric models, protection effects on import evolution. According to our results, these effects were moderate. The different behaviour of rough and manufactured timber, therefore, may be explained by differences in their competitiveness, which is related to Spanish timber resources and to the integration of the domestic timber market.

KEY WORDS: Timber, International trade, Forestry, Contemporary Spain

JEL Codes: C22, N53, N54, N73, N74 


\section{Introducción ${ }^{1}$}

L a madera siguió siendo una materia prima esencial para la economía mundial al menos hasta mediados del siglo $X X^{2}$. Si bien el uso masivo de energías fósiles hizo disminuir su importancia como combustible, la madera siguió siendo utilizada a gran escala en muchas actividades (como la minería, la construcción de tendidos ferroviarios o la construcción de edificios y obras públicas) que estaban en la base del crecimiento económico. Si a eso añadimos los nuevos usos que fueron apareciendo para ese producto desde finales del siglo XIX (pasta de madera para papel o productos químicos a través de su destilación), es fácil entender que su relevancia como materia prima no declinara ${ }^{3}$. Para el caso español, en un trabajo reciente (Iriarte y Ayuda, 2006) hemos comprobado cómo el proceso de crecimiento económico que se produjo desde finales del siglo XIX hasta la Guerra Civil, pese a estar basado en gran medida en el uso de nuevas tecnologías y de nuevos materiales, estuvo también vinculado a un consumo creciente de madera que, en términos absolutos, se triplicó entre 1860 y 1935. Paralelamente, sabemos que la madera tuvo un peso considerable en el conjunto de las importaciones españolas. Según las cifras aportadas por Prados (1988), ese producto representó un valor muy estable en el conjunto de las compras exteriores, que se situó en torno al 4,5 por 100 del total entre 1875 y 1929, a pesar de que durante esos años la composición del conjunto de las importaciones fue cambiando considerablemente. Desde esta doble perspectiva, analizar en profundidad las importaciones de un producto de consumo creciente que seguía siendo imprescindible para muchas actividades, medir la protección que se fue estableciendo al respecto y descubrir en qué medida las variaciones de esa protección fueron determinantes en la propia evolución de las compras exteriores, constituye un estudio de caso interesante que nos puede ayudar a entender mejor el papel desempeñado por las medidas arancelarias y sus consecuencias para el crecimiento económico.

[Fecha de recepción del original, agosto de 2006. Versión definitiva, mayo de 2007]

1 Este trabajo ha contado con la financiación de los proyectos de investigación del Ministerio de Educación y Ciencia "La integración internacional del sector agrario español en los siglos XIX y XX" (SEJ2005-07556) y “Validación y análisis de modelos econométricos" (BEC2003-01757). Agradecemos muy especialmente las sugerencias y la ayuda de Domingo Gallego y Vicente Pinilla. También los comentarios de los asistentes al XXI Seminario de Historia Económica de Zaragoza, en el que se expuso una versión previa del mismo, han servido para mejorarlo. Los comentarios de los cuatro evaluadores anónimos de Investigaciones de Historia Económica nos han sido igualmente de gran utilidad. Los errores que puedan subsistir son de nuestra exclusiva responsabilidad.

3 Sobre los usos declinantes y emergentes de la madera, véanse Elorrieta (1913), Wrigley (1993), Iriarte (1995), Sieferle (2001) y Zapata (2001). 
Los trabajos que hasta el momento han dedicado alguna atención específica a las importaciones españolas de madera son escasos y muestran unas interpretaciones en gran medida coincidentes. El trabajo de Gallego y Pinilla (1996) apuntaba una alta dependencia exterior maderera de España y realizaba una diferenciación básica entre las importaciones de madera labrada (muebles y otras manufacturas) y de madera sin labrar (madera en bruto y aserrada en piezas de diferente tamaño y forma), considerando a estas últimas entre las denominadas "naturales"; esto es, entre aquellas que tenían un crecimiento prácticamente constante porque no encontraban competencia en la producción interna. Posteriormente, Zapata (1998, p. 317) daba por buena esta clasificación y la utilizaba para afirmar que existía una "perfecta complementariedad entre la producción nacional y las maderas importadas". Finalmente, Iriarte (2005a) ratificaba la alta dependencia maderera exterior española situándola en perspectiva internacional $\mathrm{y}$, tras comprobar el distinto comportamiento de las importaciones según el grado de elaboración, proponía que esas diferencias podían deberse al diverso tratamiento arancelario aplicado a la madera labrada y sin labrar.

Este exiguo estado de la cuestión sugiere, sin embargo, muchas preguntas. En primer lugar, ¿a qué se debió la alta dependencia exterior maderera de España? ¿Representaron las importaciones de ese producto un valor estable respecto de su consumo interior o hubo cambios a lo largo del tiempo? En segundo lugar, habida cuenta de que durante el período considerado se produjo el conocido giro proteccionista de la economía española, ¿cómo se vio afectada la madera y cómo fueron variando sus niveles de protección? En tercer lugar ¿qué papel jugó esa protección? ¿Puede seguir afirmándose que resultó determinante a la hora de modelar las importaciones de los distintos tipos genéricos de madera, o las cosas fueron más complejas? De ser así, ¿a qué otras causas podemos atribuir el comportamiento de las importaciones?

Para contestar a estas preguntas, en la segunda sección de este trabajo se analiza el consumo español de madera en perspectiva internacional, se realiza una nueva clasificación de la madera diferenciándola entre "madera manufacturada" y "madera no manufacturada", y se mide el nivel exacto que las importaciones de cada uno de esos dos grupos representaron sobre su consumo respectivo. En la tercera sección se ofrecen dos series de protección (una para cada uno de los grupos considerados) durante el período comprendido entre 1880 y 1935, que se contrastan con algunos datos cualitativos sobre la política arancelaria aplicada a esos productos. Utilizando esas series, en las secciones cuarta y quinta se realizan sendos ejercicios econométricos, a través de los cuales se trata de determinar qué variables fueron decisivas para explicar el comportamiento de las importaciones en uno y otro caso, y se razona sobre la evolución de la competitividad de las maderas nacionales respecto de las extranjeras. Finalmente, en la sexta sección se resumen las principales conclusiones. 


\section{Consumo e importaciones}

La madera mantuvo un protagonismo elevado en los procesos de crecimiento económico vinculados a la primera y la segunda revolución industrial. Para el caso español contamos con una medición al respecto, para el período 1880-1935, que muestra cómo el consumo de madera presentó una elasticidad positiva respecto del PIB de en torno al 0,47 por 100, y cómo la demanda de madera creció tanto en la inmensa mayoría de los usos que podrían considerarse tradicionales, como en sus usos emergentes (Iriarte y Ayuda, 2006). Esta situación puede completarse y matizarse si ponemos en relación el consumo español de madera con el de otros países hacia 1929, fecha para la que se han localizado datos internacionales sobre el mismo. En este sentido, el Cuadro 1 presenta algunas cifras básicas de superficie de bosques arbolados, de producción y de consumo de madera para varios países europeos. De su lectura se pueden extraer algunas conclusiones interesantes.

Como puede observarse, el consumo de madera por habitante podía alcanzar niveles muy diferentes en los distintos países considerados ${ }^{4}$. Una parte de esas diferencias podría explicarse por la superficie de bosque arbolado existente en cada país. De hecho, si descontamos la economía británica que, como es sabido, era una gran importadora de materias primas y también de madera, en los demás casos considerados la gradación descendente del consumo por habitante coincide con una gradación, también descendente, de la superficie de bosques arbolados como porcentaje de la superficie total de cada país. En este contexto, la situación española parece similar a la de otros países mediterráneos como Italia o Grecia: los tres contaban con una amplia superficie de monte que ocupaba casi la mitad del territorio, pero también en los tres casos la superficie de bosques poblados era sensiblemente menor, estando constituida una parte muy considerable de los montes por superficie rasa o de matorral no apta para la obtención de madera. La diferencia estaba, sin embargo, en la intensidad con la que eran explotados los bosques poblados, ya que mientras Italia obtenía una cantidad de madera por hectárea similar a Francia (concentrada probablemente en la zona norte y especialmente en la alpina), España y Grecia registraban unas extracciones por hectárea mucho más exiguas.

Esa baja producción relativa de madera de los montes españoles puede explicarse por una combinación de factores históricos y ambientales. En cuanto a los primeros, conviene recordar que la ocupación humana de la Península desde épocas remotas fue haciendo disminuir las superficies arboladas de muchas zonas, dinámica que se reforzó desde el siglo XIX en un contexto de crecimiento de la población, de cambio institucional (reforma agraria liberal y, en especial, desamortizaciones) y

4 Sobre las diferencias en el consumo de madera por países, pueden consultarse, además de Lleó (1929), Brown (1919) y Arbós (1935). 


\section{CUADRO 1}

CONSUMO, SUPERFICIE Y PRODUCCIÓN DE MADERA EN VARIOS PAÍSES EUROPEOS

A FINALES DE LA DÉCADA DE 1920

\begin{tabular}{|c|c|c|c|c|c|}
\hline & (1) & (2) & (3) & (4) & (5) \\
\hline Suecia & 5,2 & n. d. & 53,6 & 35,0 & 1,5 \\
\hline Portugal & 1,6 & n. d. & 21,3 & 5,3 & 2,7 \\
\hline Francia & 0,7 & 19,6 & 18,7 & 24,2 & 2,3 \\
\hline Italia & 0,4 & 44,2 & 18,0 & 12,0 & 2,2 \\
\hline Gran Bretaña & 0,3 & n. d. & 3,9 & 1,3 & 1,1 \\
\hline España & 0,2 & 49,6 & 14,9 & 2,7 & 0,4 \\
\hline Grecia & 0,1 & 54,0 & 12,9 & 0,2 & 0,1 \\
\hline \multicolumn{6}{|c|}{ 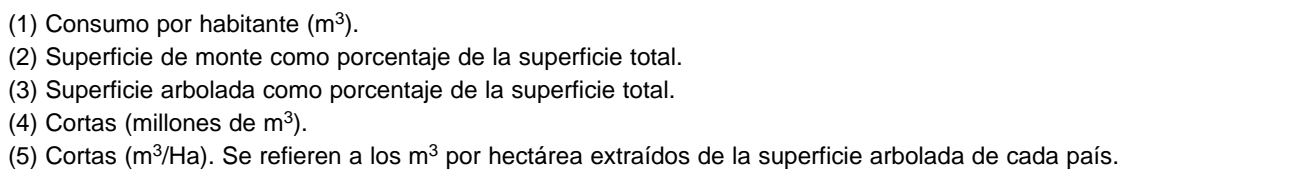 } \\
\hline \multicolumn{6}{|c|}{$\begin{array}{l}\text { Fuentes: Lleó (1929). La cifra de cortas en España ha sido corregida al alza según los datos de consumo español de } \\
\text { madera estimados por Iriarte y Ayuda (2006). Las cifras de Portugal se han corregido a la baja según los datos } \\
\text { de producción aportados para ese país por Días (2005, Anexo F, p. 575). }\end{array}$} \\
\hline
\end{tabular}

de expansión de las superficies cultivadas ${ }^{5}$. En lo que respecta a los segundos, cabe decir que las características ambientales de los bosques mediterráneos resultan mucho menos apropiadas para la obtención de madera que las de los bosques atlánticos y boreales. La escasez relativa de lluvias y el calor excesivo influyen negativamente en el crecimiento de los árboles, dando lugar, muchas veces, a ejemplares de dimensiones reducidas e irregulares en comparación con los de los boques más septentrionales. Además, los inviernos cortos y suaves hacen que los anillos de crecimiento sean menos compactos, de modo que el producto obtenido, salvo excepciones, ofrece menor resistencia y, en el contexto tecnológico de la época que nos ocupa, podía ser poco apropiado para algunos $u^{6}{ }^{6}$. De hecho, la ventaja comparativa de España en términos forestales estaba, precisamente, en productos del bosque mediterráneo como el corcho y la resina, que no sólo cubrieron el consumo interior sino que llegaron a copar, en ciertos momentos, una parte sustancial de los mercados internacionales ${ }^{7}$.

5 Sobre el proceso de deforestación se pueden encontrar referencias en Bauer (1980), Groome (1990), Gómez Mendoza (1992), y Manuel y Gil (1998). Sobre la relación entre la expansión de las roturaciones y la desaparición de superficies de monte, véase GEHR (1994).

Nájera (1948) y M’Hirit (1999).

Uriarte (1995) para la resina; Parejo (2006) para el corcho. 
A lo dicho, cabe añadir las dificultades de acceso a las principales masas maderables del país y, sobre todo, las dificultades de transporte dentro de los montes y entre éstos y los centros de consumo. Teniendo en cuenta que la red ferroviaria había sorteado los principales macizos montañosos, el transporte de madera hasta las estaciones de carga podía resultar lento y costoso. En muchas ocasiones se contaba como única alternativa con la vía acuática, que debido a su estacionalidad dificultaba el transporte y podía afectar negativamente a la calidad del producto. Las dificultades para la integración del mercado maderero que esa situación podía plantear, aunque están aún por medir, parecen evidentes ${ }^{8}$.

Esa conjunción de factores parece suficiente para explicar que la economía española presentara un bajo consumo relativo de madera y tuviera que recurrir, además, a las importaciones. Ahora bien, conviene tener en cuenta que la situación descrita no era necesariamente inmutable. Al contrario, la obtención de madera pudo ir cambiando parcialmente en función de la política forestal, de la construcción de nuevas infraestructuras o de las propias exigencias del mercado de madera que, con el tiempo, pudo ir demandando un tipo de producto en el que el tamaño y la resistencia eran menos importantes. Así pues, ¿cómo fue evolucionando la dependencia maderera exterior española?

Para contestar a esa pregunta conviene realizar antes una desagregación de las importaciones de madera en dos grandes grupos diferenciados por su grado de elaboración. Para ello hemos partido de la clasificación realizada por Gallego y Pinilla (1996), que diferenciaba entre madera labrada y madera sin labrar, pero hemos introducido en ella algunas modificaciones que, a nuestro modo de ver, la mejoran. Esas modificaciones han consistido, en lo esencial, en dar una distinta consideración a las duelas, atendiendo tanto a su bajo grado de elaboración como a los niveles arancelarios que se fueron estableciendo para ese producto concreto ${ }^{9}$. Debido a estos cambios, y para diferenciar nuestra clasificación de la de los citados autores, vamos a denominar a los grupos obtenidos como "madera manufacturada" y "madera no manufacturada"10. La evolución de las importaciones de uno y otro tipo de madera se recogen en

8 Sobre los problemas de transporte forestal, Baró (1914) y (1920).

9 Las duelas para barriles, que aparecen sistemáticamente desagregadas en las Estadísticas de comercio exterior, tenían un grado de elaboración, así como un nivel de protección arancelaria, mucho más parecidos al de las traviesas de ferrocarril o los postes (que Gallego y Pinilla incluían como madera sin labrar) que al de los muebles o los barriles ya montados (que incluían como madera labrada).

10 En nuestra clasificación, la "madera no manufacturada" incluye troncos, tablones y madera aserrada que no requería un alto grado de elaboración más allá del aserrío (traviesas, postes, duelas y madera para cajas). La "madera manufacturada", por su parte, incluye los barriles ya montados y las diferentes clases de muebles y objetos fabricados con madera. El cálculo no contabiliza las importaciones de pasta de papel, ya que no hay seguridad de que se tratara en todos los casos de pasta fabricada con madera (Zapata, 2001). Sí se han contabilizado, sin embargo, los troncos de madera que, a partir de 1906, se importaron para ese fin. Al tratar con la madera como materia prima y no como fuente de energía, tampoco se contabilizan las importaciones de leña, ni las de carbón vegetal. 


\section{CUADRO 2}

EVOLUCIÓN DE LAS IMPORTACIONES DE MADERA, 1860-1935

(a)

\begin{tabular}{rccccccrrr}
\hline & $\mathbf{( 1 )}$ & $\mathbf{( 2 )}$ & $\mathbf{( 3 )}$ & $\mathbf{( 1 )}$ & $\mathbf{( 2 )}$ & $\mathbf{( 3 )}$ & $\mathbf{( 1 )}$ & $\mathbf{( 2 )}$ & $\mathbf{( 3 )}$ \\
\hline $\mathbf{1 8 6 0 - 1 8 8 0}$ & 22.660 .706 & 2.063 .629 & 24.724 .335 & 91,7 & 8,3 & 100 & 59 & 43 & 57 \\
$\mathbf{1 8 8 1 - 1 8 9 0}$ & 38.415 .884 & 4.760 .410 & 43.176 .294 & 89,0 & 11,0 & 100 & 100 & 100 & 100 \\
$\mathbf{1 8 9 1 - 1 9 0 5}$ & 38.214 .149 & 3.336 .526 & 41.550 .675 & 92,0 & 8,0 & 100 & 99 & 70 & 96 \\
$\mathbf{1 9 0 6 - 1 9 1 3}$ & 47.342 .839 & 3.441 .119 & 50.783 .958 & 93,2 & 6,8 & 100 & 123 & 72 & 118 \\
$\mathbf{1 9 1 4 - 1 9 1 9}$ & 21.468 .568 & 2.461 .308 & 23.929 .876 & 89,7 & 10,3 & 100 & 56 & 52 & 55 \\
$\mathbf{1 9 2 0 - 1 9 2 9}$ & 52.832 .526 & 3.948 .960 & 56.781 .485 & 93,0 & 7,0 & 100 & 138 & 83 & 132 \\
$\mathbf{1 9 3 0 - 1 9 3 5}$ & 46.440 .720 & 1.418 .352 & 47.859 .072 & 97,0 & 3,0 & 100 & 121 & 30 & 111 \\
\hline
\end{tabular}

(a) Volumen de importaciones. Se ha obtenido multiplicando las importaciones de cada partida, en unidades físicas, por los precios de importación de cada partida en 1910, y se expresa en medias anuales para cada periodo.

(b) Composición en porcentaje.

(c) Números índice (base 100 = 1881-1890).

(1) Madera no manufacturada.

(2) Madera manufacturada.

(3) Total $=(1)+(2)$.

La división cronológica responde a un intento de captar los efectos sobre las importaciones de algunos de los principales cambios arancelarios (1891, 1906 y 1922), así como de los dos principales períodos de crisis de los mercados internacionales, la I Guerra Mundial y la depresión de los años treinta.

Fuentes: Estadísticas de comercio exterior de España, (1860-1935).

el Cuadro 2. El Gráfico 1, por su parte, muestra los porcentajes que las importaciones fueron representando sobre una estimación del consumo de cada uno de esos grupos ${ }^{11}$.

Como puede apreciarse, en términos generales las importaciones totales de madera tuvieron, en el largo plazo, un crecimiento moderado que sólo se rompió en el lapso posterior a 1891 (con un estancamiento a la baja) y, sobre todo, durante los años de la I Guerra Mundial (con una caída, ahora sí, significativa de las compras exteriores, de más del 40 por 100). Esa evolución vino marcada, sin embargo, por las importaciones de madera no manufacturada, que representaron en todo momento en torno a un 90 por 100 del total. Por el contrario, las importaciones de madera manufacturada, tras crecer en la década de 1880, registraron un estancamiento con tendencia a la baja que las mantuvo en niveles inferiores a los alcanzados a finales del XIX, incluso en aquellos momentos en los que invirtieron ligeramente la tendencia.

11 Sobre la obtención de la serie de consumo de madera y su fiabilidad, véase Iriarte y Ayuda (2006). 


\section{GRÁFICO 1}

PARTICIPACIÓN DE LAS IMPORTACIONES DE MADERA MANUFACTURADA

Y NO MANUFACTURADA SOBRE EL CONSUMO DE AMBOS TIPOS DE PRODUCTOS, 1881-1935

(medias móviles quinquenales centradas en el último año)

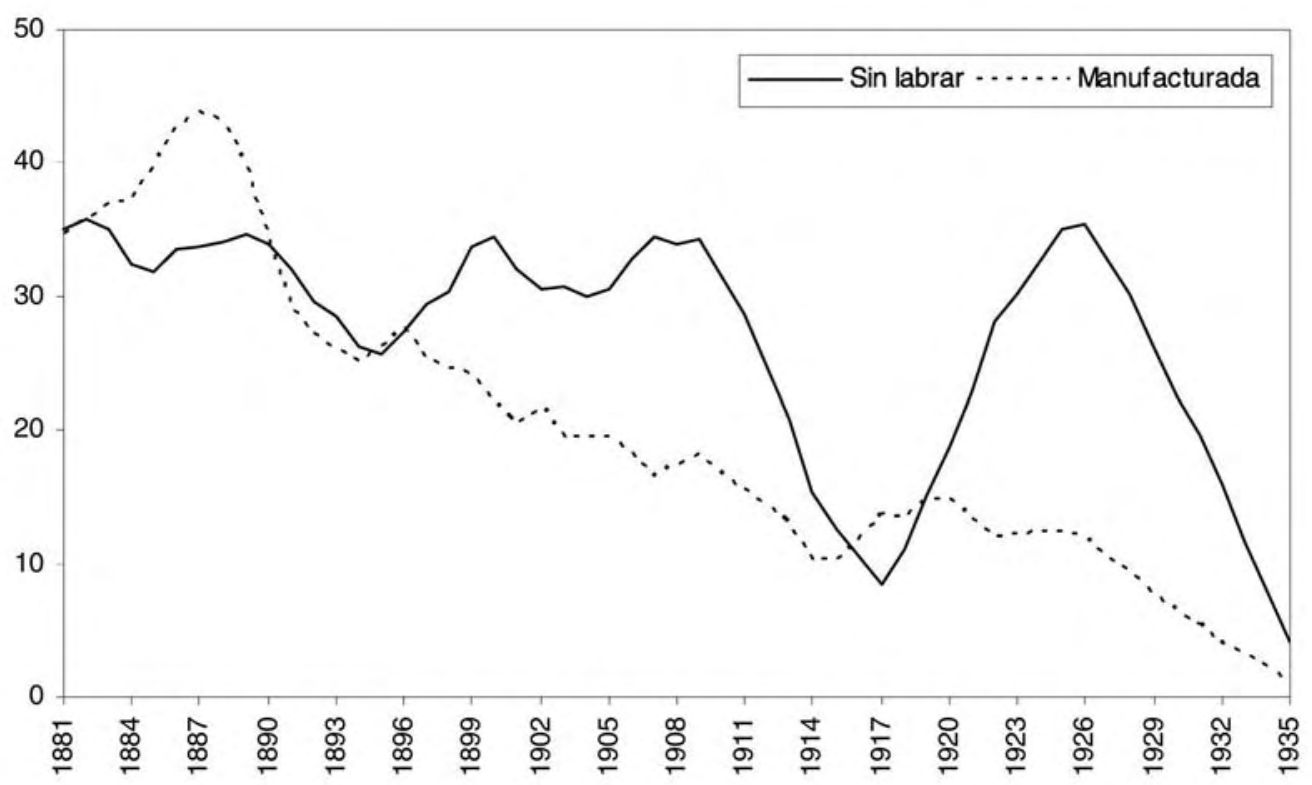

Fuentes: Para las importaciones de madera, Estadísticas de comercio exterior de España (1880-1935). Para el consumo de madera, Iriarte y Ayuda (2006).

Esta distinta evolución se detecta mucho más nítidamente poniendo en relación las importaciones con el consumo (Gráfico 1). Así, en el caso de la madera no manufacturada, las importaciones tuvieron una evolución que cabría considerar cíclica, ya que las caídas de porcentaje sobre el consumo fueron seguidas de períodos de crecimiento que volvían a situar el nivel muy cerca de la situación inicial. En este sentido, los dos momentos de mayor descenso de las importaciones respecto al consumo coincidieron con las dos grandes crisis del comercio internacional, esto es, la I Guerra Mundial y la depresión de los años treinta. Por contra, las importaciones de madera manufacturada, después de crecer en la segunda mitad del decenio de 1880, iniciaron una caída prácticamente sin freno que las acabó situando por debajo del 15 por 100, incluso en los períodos en los que se produjo algún leve repunte de los porcentajes.

En definitiva, los datos parecen hablar de un claro proceso de sustitución de importaciones en el caso de la madera manufacturada, y de una tendencia al mantenimiento de la dependencia exterior en el de la madera no manufacturada, sólo rota 
en los momentos de dificultad para adquirir ésta en otros países. En un trabajo anterior (Iriarte, 2005a), aunque de forma mucho menos evidente, se detectaban ya estas diferencias y se intentaban explicar aludiendo al tratamiento arancelario que, siguiendo criterios industrialistas, habría frenado las importaciones de madera manufacturada y habría sido permisivo con las importaciones de madera como materia prima. ¿Qué podemos avanzar en la actualidad al respecto?

\section{Una medición de la protección a la madera}

Para profundizar en esta cuestión es preciso medir la protección nominal que se fue estableciendo para cada uno de los dos tipos de madera. Y la tarea no es en absoluto sencilla si tenemos en cuenta que la forma en que se puede y debe medir la protección ha sido uno de los temas más debatidos en la historia económica española ${ }^{12}$. Por ello, quizá convenga advertir que nuestra intención no es entrar en ese debate (difícilmente podríamos hacerlo tratando sólo un producto), sino simplemente, siguiendo la idea de Pardos (2001, p. 72) de que no existe un método unánimemente reconocido para medir la protección porque todas las propuestas ofrecen sesgos de uno u otro signo, tratar de realizar una estimación correcta, que nos ofrezca pistas sobre por dónde pudieron ir las cosas. En este sentido, la principal ventaja con la que contamos es que estamos tratando con un grupo de productos relativamente homogéneo, cuyas importaciones, además, mantuvieron una estructura muy estable en el transcurso del tiempo. Como hemos visto, la madera no manufacturada acaparó en todo momento un porcentaje muy cercano al 90 por 100 del total de importaciones; pero, además, la composición interna de cada uno de los dos grupos considerados permaneció también bastante estable en el tiempo, lo que evita tener que afrontar el arduo problema de las ponderaciones y permite operar con medias simples.

Partiendo de ahí, la protección nominal se ha calculado como el porcentaje que los derechos arancelarios suponían sobre el valor de las importaciones de cada partida, introduciendo para ello varias correcciones ${ }^{13}$. La primera tiene que ver con los derechos arancelarios, que, como es sabido, vienen expresados a partir de 1906 en pesetas oro, y que se han convertido a pesetas corrientes ${ }^{14}$; la segunda concierne a las

12 Véanse Tirado (1994); Tena (1999) y (2001); y Sabaté y Pardos (2001).

13 Previamente hemos descartado la posibilidad de utilizar un indicador de protección específica (pesetas por metro cúbico de madera importada), ya que es un método poco apropiado para la madera manufacturada, cuya protección podía depender más del valor añadido por la transformación que de la cantidad de materia prima incorporada.

14 Véase Sabaté (1996). Para la conversión de pesetas oro a pesetas corrientes, Aixalá (1999). 
valoraciones oficiales, que, como también es conocido, presentan muchos problemas, habiéndose corregido por ello en función del comportamiento de los precios británicos de importación, de forma que la protección nominal refleja los derechos ingresados sobre una estimación del valor a precios de mercado ${ }^{15}$. El problema de las series así calculadas es, sin embargo, que en ellas las variaciones en la protección pueden deberse tanto a cambios en el arancel como a cambios en los precios relativos. Para evitar este problema y tratar de aislar la influencia directa del arancel, se ha efectuado una última corrección. Así, siguiendo la metodología utilizada por Gallego (2003), se han multiplicado los niveles de protección ad valorem calculados para cada tipo de madera por los respectivos índices de precios de las importaciones inglesas de ambos grupos de productos, corregidos a su vez por el de la cotización peseta/libra. De este modo, hemos obtenido lo que dicho autor denomina protección "legal"16.

Por lo demás, para ofrecer una serie continua hasta 1935, hemos tenido que estimar los derechos arancelarios cobrados en el período 1921-1935, en el cual las Estadísticas de Comercio Exterior dejaron de recoger dicho dato. Para salvar este escollo se ha recopilado información sobre las tarifas aplicadas en el arancel de 1922 a las diferentes partidas de madera (Legislación de Aduanas, 1922). Como las tarifas aplicadas a cada país podían variar de la primera a la segunda columna del arancel en función de los tratados comerciales, se ha considerado que la cláusula de nación más favorecida se aplicaba a todos aquellos países que tenían tratado comercial con España (Comisión Protectora de la Producción Nacional, 1923). Partiendo de ahí, se han calculado los derechos pagados cada año por cada partida y país para proceder, posteriormente, al cálculo de la protección ad valorem. Para ello ha sido necesaria una última corrección referida al lapso 1928-1935, ya que durante estos años las valoraciones que aparecen en las estadísticas vienen expresadas en pesetas oro, por lo que se han convertido a pesetas corrientes para dar homogeneidad a la serie ${ }^{17}$. Teniendo en cuenta todas estas correcciones, la protección referida al período 1921-1935 puede considerarse como la parte más débil de la estimación y no se descarta que pueda estar sesgada al alza. En cualquier caso, más allá del nivel concreto que alcanzara la protección en esos años, la tendencia creciente de la misma parece bastante clara.

La protección "legal", obtenida según el método descrito, aparece reflejada en el Gráfico 2 y muestra una evolución que, en sus líneas generales, es coherente con lo que sabemos sobre la política arancelaria aplicada a la madera.

\footnotetext{
15 Una forma de operar similar ha sido utilizada por Prados (1986), Tirado (1996) y Gallego (2001 y 2003).

16 El resultado del cálculo de la protección legal señalado en el texto se puede leer “... como el efecto protector de las tarifas de cada año si los precios internacionales [de los productos implicados] y la cotización de la peseta [respecto a la libra] se hubieran mantenido invariables" (Gallego, 2003, pp. 70-71). En nuestro caso, el período base para realizar el cálculo ha sido 1880-1884.

17 Véase Barciela, Giráldez, GEHR y López (2005), p. 325.
} 


\section{GRÁFICO 2}

PROTECCIÓN “LEGAL” DE LA MADERA NO MANUFACTURADA Y DE LA MADERA

MANUFACTURADA EN ESPAÑA, 1880-1935

(porcentajes ad valorem corregidos)

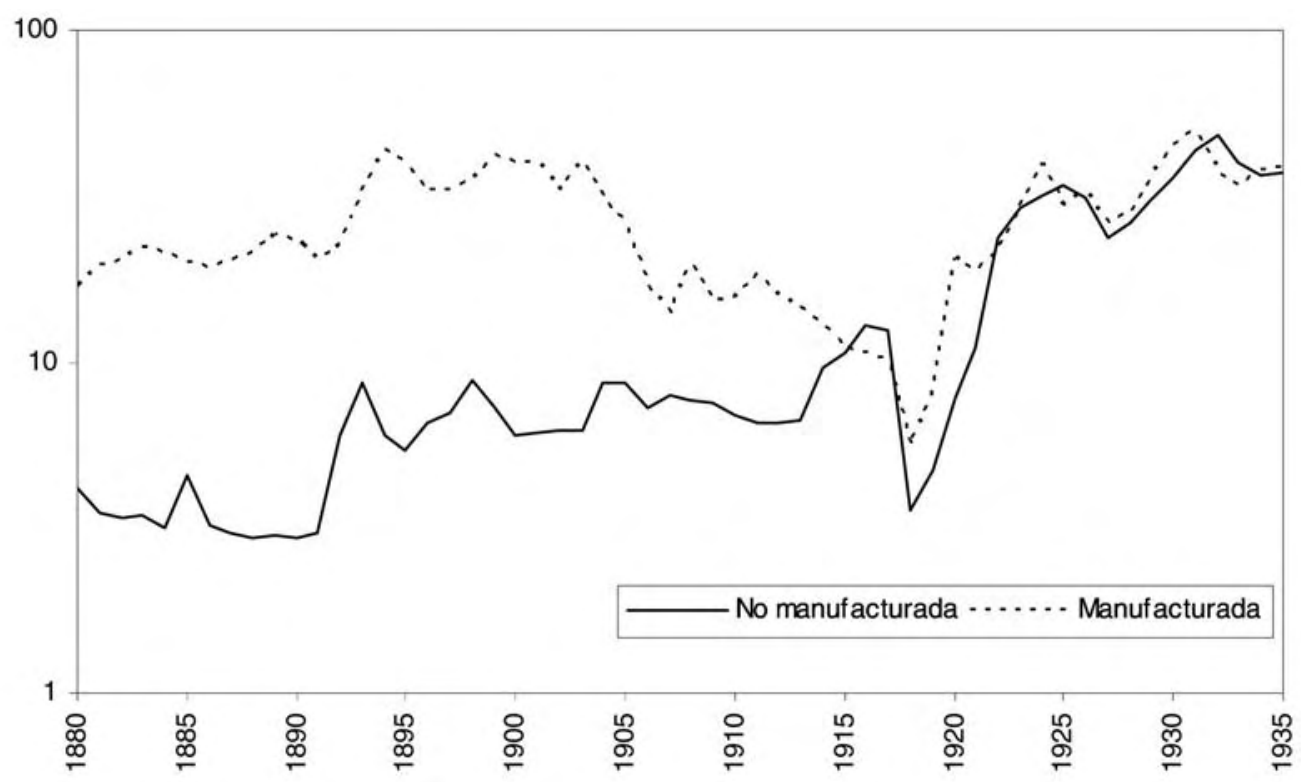

Fuentes: Estadísticas de comercio exterior de España (1880-1935), Gallego (2003), Legislación de Aduanas (1922), Comisión Protectora de la Producción Nacional (1923).

En primer lugar, se percibe claramente cómo durante los años ochenta el nivel de protección de la madera manufacturada era ya sensiblemente superior al de la madera no manufacturada, lo cual puede explicarse como consecuencia del apoyo a las industrias transformadoras proveniente del arancel Figuerola de 1869 y también de los tratados comerciales firmados en la década de 1880 con algunos de los principales países productores de madera no manufacturada, como Suecia, Rusia o Finlandia (Serrano, 1987), que debieron contribuir a mantener la protección de la madera como materia prima en unos niveles bajos. En segundo lugar, el giro proteccionista de 1891 queda perfectamente datado, con una subida de la protección a ambos tipos de madera que, además de seguir las directrices generales de la política arancelaria del momento, parece que satisfizo las peticiones recibidas sobre la madera por la comisión arancelaria en 1890 , que de uno u otro modo reclamaban una subida de la protección ${ }^{18}$. 
Posteriormente, y hasta 1913, la protección de la madera no manufacturada se mantuvo en un nivel estable, mientras que, por el contrario, la de las manufacturas de madera siguió una tendencia claramente declinante. El hecho de que el volumen de las importaciones de este último tipo de productos se hubiera reducido en más de un 50 por 100 entre 1891 y 1903 pudo estar en la base de esa relajación de los niveles de protección a partir de la segunda fecha ${ }^{19}$. Por otra parte, también la evolución de la protección a partir de 1913 parece tener su lógica. La I Guerra Mundial trastocó en profundidad los mercados internacionales de madera y generó en España una protección espontánea que, de un lado, hacía inútiles las tarifas elevadas y, de otro, incentivaba la producción interior en un momento en el que los costes de explotación y transporte resultaban prácticamente irrelevantes debido al alto precio de venta ${ }^{20}$. En este nuevo contexto, cuando las posibilidades de volver a importar madera comenzaron a recuperarse en los años veinte, los productores se fueron organizando para reclamar al Estado una elevación de la protección a todos los tipos de madera, que por lo que parece tuvo sus efectos. Entre 1921 y 1928 se volvió a los niveles de protección de preguerra, con la novedad de que ahora la protección a la madera no manufacturada (la que más había tenido que desarrollarse durante la conflagración por la imposibilidad de importar) superó ligeramente a la de la madera manufacturada. La política corporativista de la Dictadura de Primo de Rivera contribuyó, sin duda, a que las presiones de los productores encontraran eco en la Administración ${ }^{21}$. Posteriormente, la protección se reforzó durante los años comprendidos entre 1929 y 1932, en unos momentos en los que la crisis internacional contribuyó a incrementar las tensiones entre los productores y los importadores de madera en España, y en los que las peticiones para elevar la protección fueron creciendo ${ }^{22}$.

En definitiva, la protección calculada parece seguir unas líneas que resultan coherentes con los datos cualitativos disponibles sobre la evolución de la política arancelaria aplicada a la madera. Sin embargo, el disponer de dos series anuales continuas de protección permite dar un paso más, tratando de descubrir en qué medida las variaciones sufridas en esa protección entre 1880 y 1935 fueron, o no, determinantes para marcar la evolución de las importaciones. Para ello, en las dos secciones

19 Según las cifras contenidas en las Estadísticas de Comercio Exterior, las importaciones de madera manufacturada contabilizadas a precios de importación de 1910 habían pasado de 4.988 .852 pesetas en 1890 a 2.244.180 en 1903 . Sobre los cambios generados por la I Guerra Mundial en los mercados internacionales de madera, véase Iriarte (2005a). Algunas reflexiones acerca de los incentivos que la conflagración generó para la explotación forestal en España, centrado en los procesos de ordenación forestal, en Iriarte (2005b).

21 En 1923 se creó la "Agrupación Forestal y de la Industria Maderera de España" cuyo presidente, Enrique de Nárdiz, fue vocal del Consejo de Economía Nacional y miembro de la Asamblea Nacional creada por la Dictadura (Arbós, 1935).

22 Esas tensiones pueden seguirse en Arbós (1935). 
siguientes se plantean dos modelos econométricos con los que se trata de profundizar en esa cuestión y se razona, además, sobre las posibles ganancias de competitividad de las maderas españolas respecto a las de origen extranjero.

\section{Protección y competitividad de la madera no manufacturada}

En primer lugar, para tratar de medir el efecto de la protección sobre las importaciones de madera no manufacturada, hemos planteado una función de demanda de importaciones, siguiendo algunas de las sugerencias de Sawyer y Sprinkle (1999):

$$
I M n m_{t}=f\left(\text { AMnm }_{t}, \text { PIB }_{t}, \text { PESP }_{t}, \text { PINT }_{t}\right)
$$

donde la variable dependiente es el volumen de importaciones de madera no manufacturada (IMnm) y las variables independientes son la protección calculada para la madera no manufacturada (AMnm), el PIB al coste de los factores (PIB), los precios españoles de la madera no manufacturada (PESP) y los precios internacionales de la madera no manufacturada $(\mathrm{PINT})^{23}$. El modelo se limita al período comprendido entre 1880 y 1935, ya que para los años anteriores no se han localizado datos sobre la evolución de los precios interiores de la madera. En este sentido, hay que advertir que la información disponible sobre los precios para el lapso considerado es escasa y bastante problemática, lo cual, como luego se comenta, puede condicionar la solidez de los resultados de nuestra medición en lo que respecta a esta variable ${ }^{24}$.

Hemos mantenido el supuesto de que España era un país precio-aceptante en el mercado internacional de madera, lo que parece realista habida cuenta de que las importaciones españolas suponían un porcentaje muy bajo del total de madera

El volumen de importaciones se ha obtenido multiplicando las unidades físicas importadas anualmente en cada partida de madera no manufacturada por su precio de importación del año 1910. Sobre el cálculo de la serie de protección, véase la tercera sección de este artículo. Para el PIB al coste de los factores, Prados (2004). Para los precios internacionales de la madera no manufacturada se ha utilizado como indicador un índice de los precios de importación británicos de madera en bruto, obtenido de Statistical Abstract for the United Kingdom. Sobre la serie de precios españoles de la madera, véase el texto y la nota siguiente. En todas las series se ha utilizado 1910 como año base.

24 Para el período 1901-1933 hemos utilizado las series de precios de "madera elaborada de pino" aportadas por Zapata (1998), pp. 60-75, que incluyen madera de esa especie aserrada en piezas de distinto tamaño (marcos de madera, madera de hilo y madera de sierra) y que, según el análisis de este autor, presentan una homogeneidad y una continuidad suficiente como para ser empleadas. Para alargar la serie hacia atrás remontándola hasta 1880, hemos realizado una estimación a partir de los datos sobre precios de la madera de pino aportados por Sala (1997), p. 343. 


\section{CUADRO 3}

CONTRASTES DE DICKEY-FULLER Y DICKEY-FULLER AUMENTADO*

\begin{tabular}{lcccc}
\hline Variables & D-F(1) & Probabilidad & D-F(2) & Probabilidad \\
\hline IMNL & $-2,47$ & 0,12 & $-3,43$ & 0,00 \\
PIB & $-2,31$ & 0,42 & $-7,07$ & 0,00 \\
AMNL & $-1,89$ & 0,64 & $-5,30$ & 0,00 \\
PINT & $-1,86$ & 0,06 & $-4,61$ & 0,00 \\
PESP & $-6,07$ & 0,00 & - & - \\
\hline
\end{tabular}

(*) D-F(1) es el estadístico de Dickey-Fuller para contrastar la hipótesis nula de que la serie es integrada de orden $1 \mathrm{I}(1)$ frente a la alternativa de que es estacionaria; y D-F(2) contrasta si la serie es $\mathrm{I}(2)$ frente a $\mathrm{I}(1)$.

importada en los mercados internacionales ${ }^{25}$. Por otra parte, prevemos que el efecto del arancel sobre las importaciones de madera será negativo al encarecer las mismas. En lo que se refiere a la elasticidad con respecto al PIB, se espera que sea positiva, por cuanto el crecimiento económico incrementaría las necesidades de esa materia prima. En cuanto a los precios españoles de la madera, lo esperable sería un signo positivo, ya que un crecimiento de los mismos aumentaría las compras exteriores. Finalmente, lo previsto para los precios internacionales sería un signo negativo, ya que su crecimiento haría disminuir las importaciones. En cualquier caso, la influencia de los precios podría variar respecto a estas previsiones en el caso de que la producción nacional no tuviera capacidad real para sustituir a la madera comprada en el extranjero.

Las variables del modelo están en logaritmos neperianos, por lo que las estimaciones de los parámetros de posición indicarán elasticidades. Para evitar el problema de regresiones espurias, en primer lugar hemos analizado el orden de integración de las variables. Para ello, hemos utilizado el gráfico de las series, los correlogramas de las mismas y los estadísticos de Dickey-Fuller y Dickey-Fuller aumentado (DickeyFuller, 1981).

Dados los resultados de este análisis individual de las series ${ }^{26}$, se ha concluido que éstas no son estacionarias y que son integradas de orden 1, a excepción de la del precio español de la madera no manufacturada, que es estacionaria y, por ello, no

\footnotetext{
25 Véase al respecto, Iriarte (2005a), p. 25.

26 No presentamos los gráficos y correlogramas por cuestiones de espacio, pero pueden ser solicitados a los autores, tanto en este caso como en el del ejercicio del apartado siguiente.
} 


\section{CUADRO 4}

ESTIMACIÓN MÍNIMO CUADRÁTICA NO LINEAL DEL MODELO DE IMPORTACIONES

DE MADERA NO MANUFACTURADA*

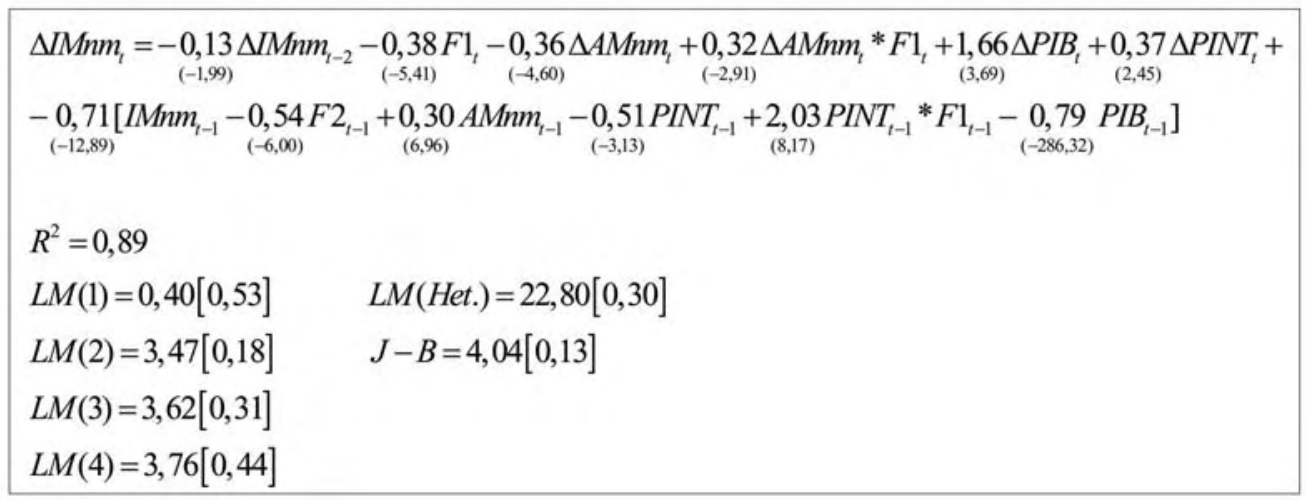

(*) Entre paréntesis aparecen los t-ratios, y entre corchetes los valores de probabilidad.

entrará a formar parte del modelo final, ya que, además, no es significativa a corto plazo incluso para niveles de significación elevados. Posteriormente, se ha analizado la posible cointegración entre las variables que son integradas de orden 1 utilizando el contraste de Engle-Granger y, aunque no es concluyente respecto a si éstas estaban o no cointegradas ${ }^{27}$, se ha seguido la metodología defendida por Kremers, Ericsson y Dolado (1992), donde se plantea un modelo en forma de mecanismo de corrección del error y, si el parámetro asociado a dicho mecanismo es significativo, se admite la hipótesis de cointegración. Por todo ello, se han planteado varios modelos en forma de mecanismo de corrección del error, y utilizando el método de mínimos cuadrados no lineales hemos estimado estos modelos teniendo en cuenta, también, dos variables ficticias: F1 (que toma valor 1 en los años 1914-1920 y 0 en el resto) con objeto de medir los efectos de la Gran Guerra y F2 (que toma valor 1 para el período 1921-1929 y 0 en el resto) para medir si hay un comportamiento distinto de las variables en los años posteriores al conflicto bélico, tanto aditivamente como multiplicativamente. Los resultados del modelo más adecuado atendiendo al criterio de selección de modelos SBIC, así como el coeficiente de determinación corregido, son los recogidos en el Cuadro 4.

El estadístico de Engle y Granger (1987) que contrasta si las 4 variables que son I(1) están cointegradas, o no, es $-3,36$, y el punto crítico para un nivel de significación del 5 por 100 es $-3,98$, lo que indica que para este nivel de significación las variables no estarían cointegradas, pero para un nivel mayor sí. 


\section{CUADRO 5}

ELASTICIDADES ESTIMADAS DEL MODELO DE IMPORTACIÓN DE MADERA NO MANUFACTURADA

\begin{tabular}{ccc}
\hline Elasticidades estimadas* $^{*}$ & Corto plazo & Largo plazo \\
\hline PIB & 1,66 & 0,79 \\
AMnm & $-0,35(-0,03)$ & $-0,30$ \\
PINT & 0,37 & $0,51(-1,51)$ \\
\hline
\end{tabular}

$\left.{ }^{*}\right)$ Entre paréntesis indicamos las elasticidades para el período 1914-1920.

En el modelo estimado en el Cuadro 4 se puede apreciar una alta significatividad del parámetro asociado al mecanismo de corrección del error, lo que nos lleva a concluir, según Kremers, Ericsson y Dolado (1992), que entre las variables importación de madera no manufacturada (IMnm), arancel de la madera no manufacturada (AMnm), PIB y precio de la madera importada (PINT) existe una relación de equilibrio estable a largo plazo.

Atendiendo a los criterios tipo LM para contrastar la hipótesis nula de no autocorrelación y de homocedasticidad, y al criterio de Jarque-Bera de normalidad, se puede concluir que el modelo no tiene problemas de autocorrelación ni de heterocesticidad, y no se rechaza la hipótesis de normalidad para un nivel de significación del 5 por 100. De las estimaciones presentadas en el Cuadro 4 podemos deducir el Cuadro 5 de elasticidades estimadas.

El modelo planteado presenta un coeficiente de determinación elevado $(0,89)$ y permite llegar a algunas conclusiones relevantes, referidas a la protección y al PIB, y a otras más débiles, concernientes a los precios. Respecto a las primeras, puede decirse que las variaciones en la protección nominal a la madera no manufacturada verificadas a partir de 1880 tuvieron en realidad unos efectos modestos (con una elasticidad aproximada del $-0,30$ por 100 a largo plazo) sobre unas importaciones que se podrían explicar, en mucha mayor medida, por la evolución del PIB. Dicho de otro modo, cuando el crecimiento del PIB tiraba de la demanda de madera como materia prima, las importaciones crecían con bastante independencia del arancel establecido (muy especialmente en el corto plazo). El comportamiento de los precios resulta, por su parte, más difícil de explicar, aunque se puede avanzar alguna hipótesis. El hecho de que los precios españoles no sean significativos puede deberse a los problemas de los datos utilizados, aunque sugiere también que buena parte de la madera importada no era sustituible por madera de origen español, debido a su calidad, a su resistencia o a sus dimensiones. En cuanto al signo positivo de los precios internaciona- 
les, podría deberse también a problemas derivados de la fuente empleada, pero viene a sugerir que las compras exteriores aumentaban incluso en momentos en los que esos precios estaban creciendo, lo cual refuerza la hipótesis de que buena parte de la madera extranjera no podía ser sustituida por madera española de igual calidad. Lo detectado para el lapso de la I Guerra Mundial indica también que esa situación tenía sus límites, de tal forma que cuando los precios internacionales traspasaban determinado umbral, su efecto sí podía tornarse negativo, aunque eso pudiera redundar en la utilización de una madera de características deficientes (Arbós, 1935, p. 298).

Ahora bien, ¿cómo encaja este hecho con el aumento de la producción interior de madera que se fue produciendo durante el primer tercio del siglo XX? ${ }^{28}$ ¿Supuso ese incremento algún avance en la competitividad de las maderas nacionales que permitiera cierto grado de sustitución de importaciones, al menos en alguna de las partidas que integran la madera no manufacturada?

Avanzar en esta cuestión resulta complicado, ya que sería preciso hacer coincidir las diferentes partidas de consumo con las de importación, lo que sólo es posible de forma parcial. De hecho, buena parte de la madera adquirida en el exterior era catalogada como "ordinaria" y se clasificaba únicamente por su grosor, sin hacer alusión al uso al que podía dedicarse, ni al tipo de madera de que se trataba, ni a su calidad. La única excepción era la madera destinada a duelas para barriles, que durante todo el período considerado se incluye de modo diferenciado. Por otra parte, a partir del arancel de 1906 aparece algún dato adicional que puede sernos de utilidad, ya que se realiza una nueva clasificación que, además de seguir desagregando la madera para duelas, hace lo mismo con la madera para traviesas de ferrocarril, para postes y para cajas; es decir, para partidas que sí pueden ponerse en relación con el consumo estimado ${ }^{29}$. Esta aproximación resulta, en todo caso, deficiente, ya que una parte desconocida de la madera que seguía entrando al mercado español como "ordinaria" podía ser, en realidad, utilizada para alguno de esos usos (o incluso para la elaboración de manufacturas) en unas proporciones que es imposible conocer. En cualquier caso, cabe suponer que la mayor parte de la madera clasificada como "ordinaria", especialmente la que venía en grandes piezas, debía de destinarse a la construcción, una actividad para la que la madera que crecía en los montes españoles no resultaba muy adecuada, tanto por sus dimensiones como por su resistencia.

28 Un análisis del incremento de la producción de madera entre 1860 y 1933, centrado en el caso de los montes públicos, en GEHR (2002).

29 Las diferentes partidas en las que puede desagregarse el consumo de madera no manufacturada, en Iriarte y Ayuda (2006). 


\section{GRÁFICO 3}

PORCENTAJES DE PARTICIPACIÓN DE LAS IMPORTACIONES DE DIVERSAS PARTIDAS DE MADERA NO MANUFACTURADA EN EL CONSUMO NACIONAL

(medias móviles quinquenales centradas en el último año)

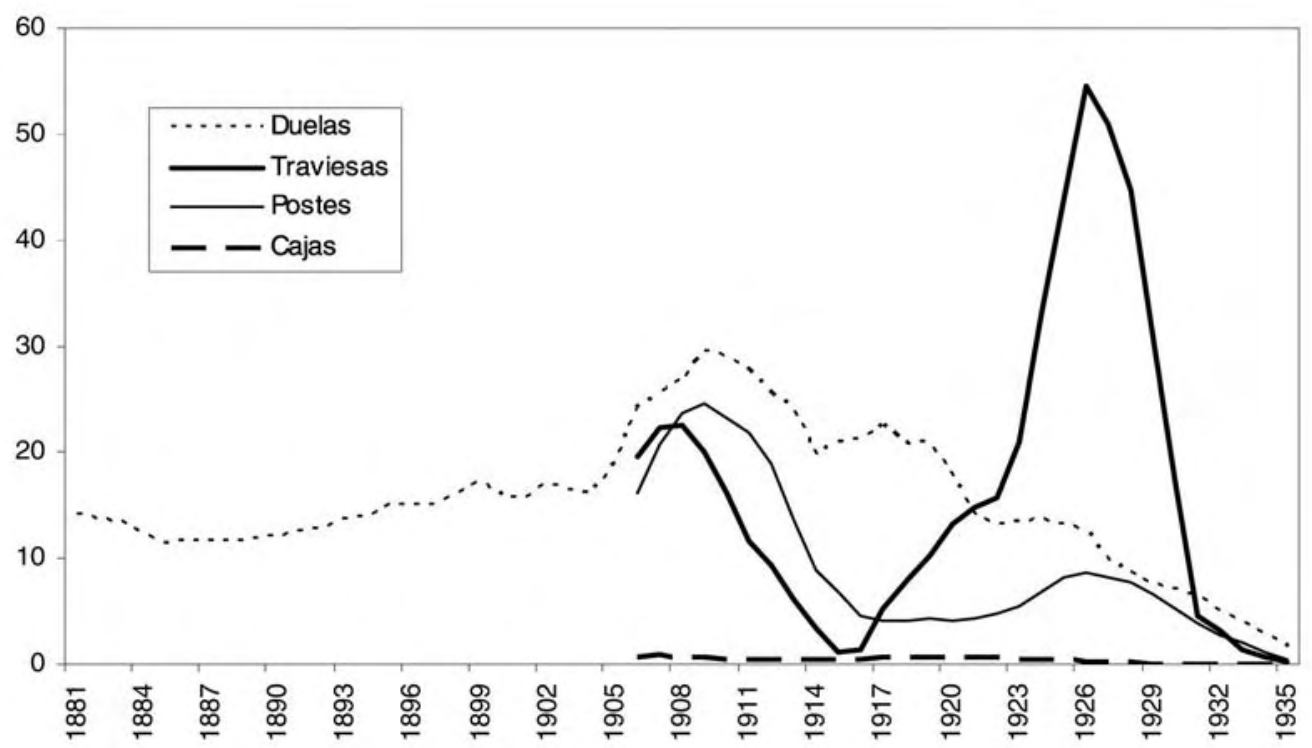

Fuentes: Para las importaciones, Estadísticas de comercio exterior de España. Para el consumo, Iriarte y Ayuda (2006).

Teniendo en cuenta las restricciones comentadas, el Gráfico 3 muestra la cuota de participación de las importaciones por partidas sobre el consumo. Como se observa, la madera importada para montar cajas alcanzó porcentajes insignificantes (apenas el 1 por 100 del consumo), lo cual sugiere que la economía española pudo ir especializándose en esa actividad, muy relacionada con la exportación de frutas, e ir cubriendo una demanda creciente sobre todo durante los años veinte. El resto de las partidas muestra una situación distinta, alcanzando en todas ellas la cuota de las importaciones sobre el consumo en 1907 porcentajes situados entre el 20 y el 30 por 100. A partir de ahí, empero, esa cuota fue decreciendo en una dinámica que, si bien parece tener un origen independiente (la reducción de las cuotas se inicia en 1909-1910), se encadenó con la caída de las importaciones forzada por la I Guerra Mundial.

Tras la guerra, tanto la madera para postes como las duelas continuaron con una tendencia descendente a largo plazo, que se reforzó aún más con la crisis de los primeros años treinta. Las traviesas, en cambio, registraron otra trayectoria: la cuota de importaciones sobre el consumo remontó incluso antes de que acabara la guerra, y subió a niveles desconocidos hasta entonces durante los años veinte. Esta evolución 


\section{CUADRO 6}

COMPOSICIÓN DE LAS IMPORTACIONES DE MADERA NO MANUFACTURADA SEGÚN SU PROCEDENCIA (porcentajes sobre el total de importaciones)

\begin{tabular}{lcccrr}
\hline & $\mathbf{1 8 8 0 - 1 8 8 3}$ & $\mathbf{1 9 0 1 - 1 9 0 3}$ & $\mathbf{1 9 1 1 - 1 9 1 3}$ & $\mathbf{1 9 2 5 - 1 9 2 8}$ & $\mathbf{1 9 3 3 - 1 9 3 5}$ \\
\hline Países Escandinavos & 29,7 & 16,6 & 24,2 & 43,8 & 45,3 \\
Rusia & 19,5 & 19,4 & 20,5 & 0,0 & 0,0 \\
Estados Unidos & 6,0 & 19,3 & 20,7 & 14,6 & 20,6 \\
Austria-Hungría & 1,8 & 2,8 & 5,3 & 0,7 & 14,0 \\
Alemania & 2,3 & 0,5 & 0,6 & 2,4 & 2,3 \\
Francia & 15,9 & 15,2 & 8,7 & 15,6 & 2,4 \\
Gran Bretaña & 0,8 & 0,8 & 0,9 & 0,3 & 0,2 \\
Italia & 16,3 & 10,8 & 2,9 & 13,6 & 1,5 \\
Portugal & 5,3 & 11,5 & 14,4 & 5,4 & 4,3 \\
Colonias españolas & 0,0 & 0,0 & 0,0 & 0,2 & 5,6 \\
Otros & 0,9 & 3,2 & 1,7 & 3,2 & 3,8 \\
\hline
\end{tabular}

Fuentes: Estadísticas del comercio exterior de España.

pudo estar relacionada con la renovación de material ferroviario acometida durante la Dictadura de Primo de Rivera; en todo caso, cesó de manera abrupta a partir de 1929. En definitiva, podría decirse que la sustitución de importaciones de madera no manufacturada se centró básicamente en algunos productos concretos como los postes y las duelas, que no requerían una materia prima de grandes dimensiones o de especial resistencia. Pero esa sustitución no tuvo la capacidad suficiente para hacer frente al incremento de la demanda de otros productos, como las traviesas de ferrocarril, que en principio presentaban unos requerimientos bastante similares.

Una segunda aproximación a la evolución de la competitividad de las maderas no manufacturadas españolas puede hacerse a través del origen de las importaciones que, indirectamente, ofrece indicios sobre la calidad de la madera importada y las posibilidades reales de ser sustituida. El Cuadro 6 recoge, para cinco períodos significativos, los porcentajes sobre el total de importaciones de madera no manufacturada aportados por cada país proveedor. Muestra una situación muy ajustada con lo que sabemos sobre los mercados internacionales de ese producto ${ }^{30}$.

30 Los cortes cronológicos escogidos para los Cuadros 6 y 7 responden a los siguientes criterios: los períodos 18801883 y 1933-1935 se han seleccionado por situarse en los extremos del lapso que nos ocupa, y los intermedios (19011903, 1911-1913 y 1925-1928), por representar momentos de repunte de las importaciones sobre el consumo (ver Gráfico 1) que resultan apropiados para la comparación. Los tres últimos permiten, además, intuir los efectos no inmediatos, pero sí de medio plazo, respectivamente, de los aranceles de 1891, 1906 y 1922. 
La preponderancia prácticamente constante de los países escandinavos; la importancia de las maderas rusas hasta su desaparición de los mercados internacionales tras la revolución de 1917; la paulatina pero constante irrupción de las maderas americanas desde principios del siglo $X X$; el ligero aumento de las maderas de Austria-Hungría, su eclipse tras la Gran Guerra y la desmembración del Imperio, y su reaparición en los años treinta como importaciones provenientes de alguno de los nuevos países (en especial, Yugoslavia); o la creciente relevancia desde los años treinta de las maderas tropicales procedentes de las colonias (en el caso español, de Guinea), vinculada con los inicios de la fabricación de tableros de madera contrachapada, son rasgos que afectaron no sólo a España, sino al conjunto de los mercados internacionales ${ }^{31}$. Al respecto, la principal peculiaridad del caso español estuvo en las compras realizadas a países vecinos como Francia y Portugal, que en algunos períodos alcanzaron cuotas bastante significativas.

Pero lo importante para lo que aquí interesa es que el origen de las importaciones presentaba una estrecha relación con la calidad de las maderas. De hecho, la madera de frondosas (principalmente roble) procedente de Estados Unidos o de Austria-Hungría tenía una calidad muy superior a la española, resultando por ello insustituible. Lo mismo ocurría, también, con buena parte de la madera de pino, que, aun siendo la más abundante en los montes españoles, tenía menor calidad que la importada. Así, por ejemplo, las maderas de los países escandinavos estaban compuestas principalmente de "pino rojo", que era muy apropiado para la construcción y la carpintería y ofrecía una calidad muy difícil de encontrar en otros mercados, e imposible de obtener en la inmensa mayoría de los bosques del país (Arbós, 1935). Algo similar cabe decir de las maderas rusas, de características naturales muy parecidas a las anteriores, si bien el tratamiento mucho más imperfecto que recibían en su proceso de explotación y transporte podía hacerlas algo menos apreciadas (Áström, 1987). Y lo mismo vale para las importaciones procedentes de Estados Unidos, que incluían, además de madera de roble, pinos grandes y resistentes (Tea, Montana, Americano) imposibles de conseguir en Europa ${ }^{32}$.

Así pues, las características naturales y la calidad derivada de ellas hacían que una parte considerable de la madera importada fuera insustituible. La excepción estaría en las maderas adquiridas en Francia y Portugal que, en general y según opinaba uno de los principales expertos madereros de España en los años treinta (Arbós, 1935), no presentaban ventaja alguna sobre las del país y eran perfectamente sustituibles por éstas. Esta idea se confirma, además, si tenemos en cuenta que los

32 La revista Montes e Industria, números 66 (enero de 1926) a 85 (agosto de 1927), ofrece pistas interesantes sobre las características de las maderas importadas en función de los países de origen. 


\section{CUADRO 7}

PARTICIPACIÓN DE LAS IMPORTACIONES DE FRANCIA Y PORTUGAL EN

EL CONSUMO ESPAÑOL DE MADERA NO MANUFACTURADA

\begin{tabular}{lccccc}
\hline & $\mathbf{1 8 8 0 - 1 8 8 3}$ & $\mathbf{1 9 0 1 - 1 9 0 3}$ & $\mathbf{1 9 1 1 - 1 9 1 3}$ & $\mathbf{1 9 2 5 - 1 9 2 8}$ & $\mathbf{1 9 3 3 - 1 9 3 5}$ \\
\hline Francia & 4,1 & 6,5 & 2,9 & 9,2 & 0,3 \\
Portugal & 1,3 & 4,9 & 4,8 & 3,2 & 0,6 \\
Ambos países & 5,4 & 11,4 & 7,7 & 12,4 & 0,9 \\
\hline
\end{tabular}

Fuentes: Estadísticas del comercio exterior de España.

dos países citados acaparaban una porción notable de las importaciones de madera para postes, traviesas y cajas, es decir, de los productos que fueron objeto de una cierta sustitución de importaciones. Por tanto, el seguimiento de lo que ocurrió con la madera procedente de Francia y Portugal puede dar algún indicio, si bien indirecto, de los posibles avances de la competitividad de las maderas españolas. A tal fin, el Cuadro 7 recoge las cifras de participación de las importaciones que provenían de ambos países en el consumo español de madera no manufacturada.

De su lectura podría concluirse, en primer lugar, que el giro arancelario de 1891 no tuvo efectos duraderos sobre la competitividad de las maderas españolas, en la medida en que la cuota sobre el consumo de las maderas francesas y portuguesas, lejos de disminuir, aumentó en los primeros años del siglo XX. Esta situación se fue alterando de manera significativa en los años siguientes; de modo que, en vísperas de la I Guerra Mundial, la dependencia maderera en relación a ambos países se había reducido sensiblemente. Sin embargo, con el auge importador de los años veinte, la cuota conjunta volvió a elevarse, guiada ahora por un fuerte repunte de las maderas procedentes de Francia, que debió de estar provocado por la fuerte devaluación del franco en el período posterior a la guerra. Finalmente, la contención de las importaciones generada por la depresión de los años treinta sí resultó decisiva para rebajar a mínimos la participación de las maderas de ambos países.

En resumen, si retomamos las dos últimas mediciones realizadas, podría decirse que la capacidad de la madera no manufacturada española para competir con la extranjera en el mercado interior existió, aunque no fue demasiado consistente. Esa situación se explica con facilidad para una parte significativa de las compras exteriores, constituida por maderas de una calidad imposible de obtener en los montes españoles, que resultaban insustituibles y que no entraban en competencia directa con la producción interior. También se importaban, no obstante, maderas de menor calidad que sí podían obtenerse en los montes españoles. Esta porción realmente sus- 
tituible es, por el momento, imposible de medir con exactitud, aunque podemos realizar una aproximación a la misma. Si aceptamos que España tenía capacidad para producir toda la madera necesaria para duelas, postes, traviesas y cajas que consumía, podemos decir que, como media para el período 1906-1935, las importaciones de madera no manufacturada podían haberse reducido en un 14 por 100 anual. Si complementariamente consideramos que se podía haber obtenido en los montes del país toda la madera que provenía de Francia y Portugal, entonces, en los lapsos de mayor auge importador, las compras exteriores podían haberse reducido en torno a un 25 por 100 anual (ver Cuadro 6).

En definitiva, lo visto hasta aquí permite una doble interpretación. De un lado, la sustitución de importaciones detectada sugiere un avance en la producción interior de madera, sobre todo desde principios del siglo XX. Esto ya se había comprobado para los montes públicos (GEHR, 2002), pero a la luz de los datos aportados podría añadirse, a modo de hipótesis, que el aumento de la producción pudo acaecer también en los montes de particulares, y quizá principalmente en los situados en la franja atlántica del país, que contaban con condiciones apropiadas para el desarrollo de especies de crecimiento rápido, adecuadas para la obtención de algunos productos cuya demanda estaba creciendo (cajas y postes, en especial). Pero, por otro lado, hay indicios de que la sustitución de importaciones podría haber sido mayor. En el contexto técnico del período considerado, la única manera de incrementar la productividad forestal pasaba por realizar cuantiosas inversiones en los montes y en las infraestructuras de acceso a los mismos, que abaratasen los costes de explotación y de transporte $^{33}$. Y, por lo que parece, las medidas para fomentar la producción y la integración del mercado, o no fueron las más apropiadas, o no tuvieron la intensidad suficiente como para lograr mejores resultados ${ }^{34}$. Los informes emitidos por algunas asociaciones forestales a finales de los años veinte así lo sugieren. Por ejemplo, la "Agrupación Forestal y de la Industria Maderera de España" aludía a la permisividad de la política arancelaria respecto a la madera no manufacturada, pero se refería también a la falta de inversiones que facilitaran la explotación de los montes y a las elevadas (y desiguales, según trayectos) tarifas ferroviarias que dificultaban una mayor fluidez en el mercado nacional maderero. $Y$, curiosamente, los importadores, organizados por su parte en la "Asociación Española de Importadores de Maderas", defendían la continuidad de las compras exteriores con argumentos muy similares, que según ellos tenían como principal consecuencia que, a igual calidad, las maderas españolas llegaran a los centros de consumo a precios más altos que las importadas.

\footnotetext{
33 Sobre está afirmación, puede verse el análisis del sector forestal estadounidense realizado por Libecap y Johnson (1979).

34 Sobre las ordenaciones forestales, véase Iriarte (2005b).
} 


\section{La sustitución de importaciones de madera manufacturada}

En el caso de la madera manufacturada, la situación fue bastante diferente ya que, como hemos visto, desde principios de la década de 1890 se fue produciendo un claro proceso de sustitución de importaciones (Cuadro 2 y Gráfico 1). Sin embargo, también aquí parece pertinente tratar de llegar un poco más lejos, preguntándonos en qué medida la explicación de esa sustitución recae en el arancel. Para ello hemos planteado un modelo similar al realizado en la sección anterior, en el que la variable dependiente es ahora la importación de madera manufacturada (IMm) y las variables independientes son el arancel calculado para la madera manufacturada (AMm), el PIB al coste de los factores (PIB) y el precio internacional de la madera manufacturada (PINTm) ${ }^{35}$. No hemos conseguido una información lo suficientemente sólida como para integrar los precios nacionales de la madera manufacturada en este ejercicio, lo cual debilita su potencialidad explicativa. Para contar con una variable adicional, hemos integrado la producción española de madera manufacturada (PMm) que nos puede dar una idea de su relación con las importaciones ${ }^{36}$. El modelo, además, introduce dos variables ficticias: F1, que recoge los efectos de la Gran Guerra y toma valor 1 para los años 1917-1919 y 0 en el resto; y F2, que toma valor 1 para los años 1922-1929 y 0 para el resto, para medir posibles cambios en los coeficientes en los años inmediatamente posteriores al período bélico ${ }^{37}$.

El signo esperado de las elasticidades de la importación de madera manufacturada con respecto a las variables explicativas es el mismo que en el caso de la madera no manufacturada. En cuanto a la producción interior, que no se incluía en el modelo anterior, esperamos que tenga signo negativo, ya que su crecimiento haría disminuir las compras exteriores.

Utilizando de nuevo los gráficos, correlogramas y estadísticos de Dickey-Fuller (Cuadro 8), se puede concluir que las series son integradas de orden 1, para un nivel de significación del 5 por 100. Utilizando los contrastes de Engle-Granger parece que existe cointegración entre las variables ${ }^{38}$.

35 El volumen de importaciones se ha obtenido multiplicando las unidades físicas importadas anualmente en cada partida de madera manufacturada por su precio de importación del año 1910. Sobre el cálculo de la serie de protección, véase la tercera sección de este artículo. El PIB al coste de los factores ha sido tomado de Prados (2004). Para los precios internacionales de la madera manufacturada se ha utilizado como indicador un índice de los precios de importación británicos de madera manufacturada, obtenido de Statistical Abstract for the United Kingdom. En todas las series se ha empleado 1910 como año base.

36 La producción de madera manufacturada se ha calculado a partir de la estimación del consumo (producción estimada = consumo - importaciones + exportaciones). Sobre la estimación del consumo, véase Iriarte y Ayuda (2006). Estas variables han sido elegidas después de probar varias, con objeto de mejorar las propiedades estadísticas del modelo.

38 El estadístico de Engle y Granger para contrastar la hipótesis nula de no cointegración frente a cointegración entre las variables es: E-G (IMm, PIB, AMm, PINTm, PMm) = -5,12 (-4,76). Entre paréntesis indicamos el punto crítico para un nivel de 5 por 100 de significación, por lo que se rechazaría la hipótesis nula de no cointegración para ese nivel de significación. 


\section{CUADRO 8}

CONTRASTES DE DICKEY-FULLER Y DICKEY-FULLER AUMENTADO*

\begin{tabular}{lcccc}
\hline Variables & D-F(1) & Probabilidad & D-F(2) & Probabilidad \\
\hline IMNL & $-0,09$ & 0,99 & $-8,73$ & 0,00 \\
PIB & $-2,31$ & 0,42 & $-7,07$ & 0,00 \\
AMNL & $-1,82$ & 0,36 & $-6,79$ & 0,00 \\
PINT & $-3,08$ & 0,12 & $-9,33$ & 0,00 \\
PESP & $-2,86$ & 0,06 & $-8,76$ & - \\
\hline
\end{tabular}

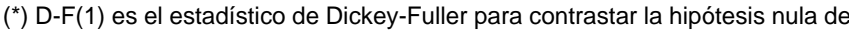
que la serie es integrada de orden $1 \mathrm{I}(1)$ frente a la alternativa de que es estacionaria; y D-F(2) contrasta si la serie es $\mathrm{I}(2)$ frente a I(1).

Planteamos varios modelos en forma de mecanismo de corrección del error para explicar las importaciones de madera manufacturada, y atendiendo a la significatividad del parámetro del mecanismo de corrección del error (-0.58) y del resto de variables, así como a los criterios de selección de modelos, AIC y SBIC, el modelo considerado más adecuado es el que aparece en el Cuadro 9.

En el modelo elegido no aparece la variable precios internacionales de la madera manufacturada (PINTm) porque no es significativa ni a corto ni a largo plazo, ni siquiera para niveles de significación altos. Además, al introducirla, los criterios de selección de modelos AIC y SBIC empeoran (también hay que tener en cuenta que para un nivel de significación del 10 por 100 esta variable, según el criterio de Dickey-Fuller, sería estacionaria). Por lo demás, el modelo no presenta problemas ni de autocorrelación ni de heterocedasticidad y no rechazamos la hipótesis de normalidad incluso para niveles de significación altos. Las estimaciones de las elasticidades se resumen en el Cuadro 10.

Como en el modelo de las importaciones de madera no manufacturada, la significatividad y el signo negativo del coeficiente del mecanismo de corrección del error (Kremers, Ericsson y Dolado, 1992) indican que las variables importación de madera labrada, su arancel y la producción interior de la misma están cointegradas.

En este caso, la capacidad explicativa del modelo (con un coeficiente de determinación de 0,74 ) es menor que en el de la madera no manufacturada. Esto puede deberse, entre otras causas, a la imposibilidad de integrar los precios nacionales por no existir información sobre ellos, y obliga a ser muy cautos con las conclusiones que siguen, que deben juzgarse como hipótesis provisionales. De cualquier modo, en el largo plazo, todas las variables significativas consideradas muestran el signo esperado, y una de las hipótesis que se deriva de los resultados es novedosa: la influen- 


\section{CUADRO 9}

ESTIMACIÓN MÍNIMO CUADRÁTICA NO LINEAL DEL MODELO DE IMPORTACIONES DE MADERA MANUFACTURADA*

$$
\begin{aligned}
& \Delta I M m_{t}=\underset{(-6,25)}{0,59} F 3_{t}+\underset{(4,71)}{1,22 \Delta A M m_{t} F 1_{t}}-\underset{(-3,38)}{0,62 \Delta P M m_{t}+} \\
& +\underset{(2,93)}{2,21} \Delta P I B_{t}-\underset{(-7,38)}{0,56\left[I M m_{t-1}\right.}-\underset{(-5,86)}{24,98}-\underset{(-3,57)}{0,63} F 2_{t-1}+ \\
& +\underset{(2,10)}{0,23} \mathrm{AMm}_{t-1}+\underset{(2,04)}{\left.0,50 \mathrm{PMm}_{t-1}\right]} \\
& R^{2}=0,74 \\
& \operatorname{LM}(1)=0,02[0,89] \quad \operatorname{LM}(\text { Het } .)=15,92[0,46] \\
& \operatorname{LM}(2)=2,42[0,30] \quad J-B=1,28[0,52] \\
& \operatorname{LM}(3)=2,60[0,46] \\
& \operatorname{LM}(4)=3,13[0,54]
\end{aligned}
$$

$\left({ }^{\star}\right)$ Entre paréntesis se encuentran los t-ratios y entre corchetes los valores de probabilidad.

\section{CUADRO 10}

ESTIMACIONES DE LAS ELASTICIDADES DE LAS IMPORTACIONES DE MADERA MANUFACTURADA

\begin{tabular}{ccc}
\hline Elasticidades estimadas & Corto plazo & Largo plazo \\
\hline PIB & 2,21 & - \\
AML & $1,22^{\star}$ & $-0,23$ \\
PML & $-0,62$ & $-0,50$ \\
\hline
\end{tabular}

(*) Sólo para los años 1917-1919.

cia de las variaciones del arancel producidas a partir de 1880 (con una elasticidad aproximada del -0,23 por 100) resultó incluso algo menor que en el caso de la madera no manufacturada; es decir, tuvo también unos efectos bastante moderados que no pueden explicar por sí solos la sustitución de importaciones observada más arriba. De hecho, la evolución de la producción nacional (con una elasticidad de -0,50) parece jugar un papel más importante a la hora de explicar la caída de las importaciones. 
Esta hipótesis, pese a su carácter provisional, puede defenderse por varios motivos. En primer lugar, debe tenerse en cuenta que el modelo planteado toma en consideración las variaciones del arancel en el tiempo, pero no el nivel arancelario concreto de partida. Este hecho resulta relevante al respecto, ya que en el primer año considerado (1880) la protección calculada para las manufacturas de madera era ya sensiblemente superior a la de la madera no manufacturada (Gráfico 2). Ello sugiere que el origen de la sustitución de importaciones pudo estar en el nivel arancelario establecido antes de 1880, probablemente desde 1869. En segundo lugar, conviene no olvidar que las manufacturas representaban un volumen reducido del total de importaciones de madera (Cuadro 2) y que, en consecuencia, su sustitución podía ser más factible que la de la parte mayoritaria. Además, y con más importancia, cabe argüir que desarrollar la producción de manufacturas de madera era más sencillo que fomentar la producción de madera en bruto en los montes españoles. De hecho, las inversiones precisas para poner en marcha serrerías o pequeñas fábricas de muebles no parece que fueran muy elevadas, debiendo de ser asumibles por pequeños y medianos capitales privados. Asimismo, la tecnología necesaria era relativamente sencilla de adquirir y su difusión debió de verse favorecida, además, por la aparición de las sierras eléctricas. Finalmente, las manufacturas de madera podían utilizar como materia prima no sólo madera nacional, sino también parte de la madera no manufacturada procedente del extranjero, lo cual aseguraría el suministro a precios asequibles. Esta situación resulta, además, coherente con el hecho de que los precios internacionales de la madera manufacturada no resultaran significativos a la hora de importar, y sugiere que los precios españoles (de los que no se han encontrado datos) debieron de ser más bajos que los internacionales, quizá por el coste más reducido de la mano de obra en España.

En todo caso, lo anterior cuadra bastante bien con los datos que conocemos sobre el crecimiento de las industrias de la madera en el conjunto del país (Nadal, 1987; Zapata, 2001) o en algunas regiones concretas (Nadal, 1990; Garrués, Hernández y Martín, 2003). La creciente concentración de las industrias de aserrado en el norte, con Galicia a la cabeza, detectada por Zapata (2001, p. 331) refuerza también la hipótesis comentada más arriba sobre la especialización maderera de esa zona desde principios del siglo XX. En definitiva, parece que las manufacturas de madera españolas fueron capaces de ganar competitividad sobre las extranjeras en el mercado interno, expulsando a la mayor parte de unas importaciones que quedaron reducidas a muebles muy específicos, probablemente de lujo, que no se fabricaban en el país. 


\section{Conclusiones}

La economía española fue aumentando su consumo tanto de madera manufacturada como de madera no manufacturada entre 1880 y 1935, aunque el recurso a las importaciones varió considerablemente en un caso y en otro. La madera manufacturada fue objeto de una clara sustitución de importaciones, que situó las compras exteriores en unos porcentajes muy bajos respecto al consumo. Por el contrario, las importaciones de madera no manufacturada (que suponían la mayor parte del total) se mantuvieron en un nivel elevado, que sólo se redujo con contundencia en aquellos momentos en los que existieron graves problemas para adquirir ese producto en los mercados internacionales. Sin embargo, la cuantificación de la protección aplicada a ambos tipos de madera y la medición de la elasticidad de las importaciones respecto a las variaciones en dicha protección permiten concluir que, en el período considerado, la responsabilidad directa de los aranceles en esa evolución de las compras exteriores fue muy moderada, lo que obliga a buscar otras causas explicativas.

La sustitución de importaciones de madera manufacturada desde finales del siglo XIX podría explicarse, en parte, por una situación heredada (el elevado nivel arancelario establecido para esos productos antes de 1880) pero, sobre todo, por la capacidad de la industria transformadora nacional de incrementar su competitividad respecto a las manufacturas extranjeras de madera. Este aumento pudo deberse a las limitadas barreras de entrada al sector (de capital y tecnológicas) y, probablemente, también a los precios más moderados de la mano de obra en España. En lo que se refiere a la madera no manufacturada, una parte considerable de las importaciones era imposible de sustituir por sus dimensiones, su calidad o su resistencia. Los resultados obtenidos sobre la influencia de los precios, pese a los problemas comentados, apuntan en esa misma dirección. Sin embargo, hay indicios que permiten afirmar que la economía española podría haber sustituido otra parte de las importaciones de madera no manufacturada, obteniéndola en el interior del país. Como aproximación, cabe señalar que las importaciones anuales podrían haberse reducido, en algunos momentos, entre un 14 por 100 (si consideramos como sustituible la madera importada para duelas, postes, traviesas y cajas) y un 25 por 100 (si consideramos como sustituible, además, la madera importada de Francia y Portugal).

Desde esta perspectiva, cabe suponer que las posibilidades de actuación de los responsables de la política que afectaba a la madera se situarían entre dos polos: o bien trataban de sustituir una parte mayor de las importaciones, invirtiendo más recursos en una política de fomento e integración del mercado maderero nacional, o bien adoptaban variaciones poco drásticas que permitieran seguir importando, aunque sin renunciar a una cierta elevación de los aranceles que 
satisficiera, al menos nominalmente, las demandas que iban en esa dirección. Según los resultados obtenidos en este trabajo, la opción elegida se acercó más al segundo polo que al primero, lo que puede reputarse coherente con la situación de un país que no estaba especialmente dotado para la producción de madera y que no se caracterizaba precisamente por la abundancia de recursos públicos. Cabe añadir, sin embargo, que esa opción no estaba exenta de problemas. De un lado, obligaba a seguir importando madera de características similares a la del país, lo que contribuía a aumentar un déficit comercial ya de por sí elevado; de otro, como señalaba el propio Arbós (1935, p. 173), implicaba un cierto sacrificio del sector maderero extractivo (no así del de las manufacturas de madera). Además, y en estrecha relación con lo anterior, la opción tomada sugiere una despreocupación implícita por la viabilidad económica de muchas zonas de montaña que podían ocupar una extensión considerable del territorio, y que podrían haberse visto más favorecidas si se hubiera potenciado en mayor medida una explotación regular y racional de sus montes maderables. Finalmente, en aquellos momentos en los que el recurso a los mercados internacionales resultaba problemático o inviable, las posibilidades que se abrían resultaban un tanto funestas: o bien se reducía drásticamente el consumo de madera (cosa que no ocurrió), o bien se echaba mano de manera improvisada de unos montes no preparados para una explotación regular, que en esas condiciones pudieron ser objeto de una sobreexplotación considerable.

\section{Bibliografía}

AIXALA, José (1999): La peseta y los precios: un análisis de largo plazo (1868-1995), Zaragoza, Prensas Universitarias de Zaragoza.

ARBÓS, José (1935): Los problemas de la madera: su importancia en España, cómo se han tratado y sus relaciones con nuestro comercio exterior, Barcelona, Herederos de Serra y Rusell.

ÁSTRÖM, Sven E. (1987): “North-eastern Europe's Timber Trade between the Napoleonic and Crimean Wars: A Preliminary Survey", The Scandinavian Economic History Review, 35, 2, pp. 170-177.

BARCIELA, Carlos; GIRÁLDEZ, Jesús; GEHR, y LÓPEZ, Inmaculada (2005): “Sector agrario y pesca", en CARRERAS, Albert, y TAFUNELL, Xavier (coords.), Estadísticas históricas de España, siglos XIX y XX, Bilbao, Fundación BBVA, pp. 245357.

BARÓ, Fernando (1914): Estudio y construcción de vías de transporte aplicadas a la saca de los productos de los montes, Madrid, Imprenta Alemana. 
-(1920): "Los transportes forestales en España: su importancia económica y social", Primer Congreso Nacional de Ingeniería, Sección $8^{a}$, Industria Forestal y sus derivadas, Madrid, Sucesores de Rivadeneyra.

BAUER, Eric (1980): Los montes de España en la Historia, Madrid, Ministerio de Agricultura, Pesca y Alimentación.

BROWN, Nelson C. (1919): Forest products their manufacture and use: embracing the principal commercial features, Boston, John Wiley \& Sons.

COMISION ARANCELARIA (1890): La reforma arancelaria y los tratados de comercio, Madrid, Sucesores de Rivadeneyra.

COMISIÓN PROTECTORA DE LA PRODUCCIÓN NACIONAL (1923): Régimen mercantil internacional de España, Madrid, Imprenta de Ramona Velasco.

DIAS, Amélia (2005): O impacto das florestas no crecimiento económico moderno durante o Estado Novo (1930-1974), Tesis Doctoral, Instituto Superior de Economía e Gestao, Universidade Técnica de Lisboa.

DICKEY, David A., y FULLER, Wayne A. (1981): "Likelihood ratio tests for autoregressive time series with a unit root", Econometrica, 49, pp. 1.057-1.072.

ELORRIETA, Octavio (1913): Evolución de la economía forestal, Madrid, Imprenta J. Laguna.

ENGLE, Robert F., y GRANGER, Clive W. J. (1987): “Cointegration and error-correction: representation, estimation and testing", Econometrica, 55, pp. 251-276.

Estadísticas del comercio exterior de España, 1880-1935.

GALLEGO, Domingo (2001): "De los limitados efectos de la política arancelaria sobre las orientaciones productivas del sector agrario español, (1869-1914)", en SUDRIÀ, Carles, y TIRADO, Daniel (eds.), Peseta y protección. Comercio exterior, moneda y crecimiento económico en la España de la Restauración, Barcelona, Edicions UAB, pp. 45-70.

-(2003): "Los aranceles, la política de comercio exterior y la estabilidad de la agricultura española (1870-1914)", Revista de Estudios Agrosociales y Pesqueros, 198, pp. 9-74.

GALLEGO, Domingo, y PINILLA, Vicente (1996): “Del librecambio matizado al proteccionismo selectivo: el comercio exterior de productos agrarios y alimentos en España entre 1949 y 1935", Revista de Historia Económica, XIV, 2, pp. 371-420.

GARRUÉS, José Ángel; HERNÁNDEZ, Salvador, y MARTÍN, Manuel (2003): “Compañías y empresarios de la madera en Andalucía: una aproximación sectorial a partir de las anotaciones registrales", en MARTÍN, Manuel, J. A.; GARRUÉS, José Ángel, y HERNÁNDEZ, Salvador (eds.), El registro mercantil: una fuente para la historia económica, Granada, Universidad de Granada, pp. 239-283.

GEHR (1994): "Más allá de la «propiedad perfecta». El proceso de privatización de los montes públicos españoles (1859-1926)" , Noticiario de Historia Agraria, 8, pp. 99-152. 
-(2002): "Política forestal y producción de los montes públicos españoles. Una visión de conjunto, 1861-1933", Revista de Historia Económica, XX, 3, pp. 509-541. GÓMEZ MENDOZA, Josefina (1992): Ciencia y politica de los montes españoles, Madrid, Ministerio de Agricultura, Pesca y Alimentación e ICONA.

GROOME, Helen J. (1990): Historia de la política forestal en el Estado español, Madrid, Agencia de Medio Ambiente.

IRIARTE, Iñaki (1995): Privatización, particularización y gestión de los montes públicos. Navarra 1855-1935, Tesis Doctoral, Universidad de Zaragoza

-(2005a): "La inserción internacional del sector forestal (1849-1935)", Revista de Historia Industrial, 27, pp. 13-47.

-(2005b): "Las ordenaciones forestales en las primeras décadas del siglo XX: cambio institucional y resultados productivos", Revista de Historia Económica, XXIII, 2, pp. 299-334.

IRIARTE, Iñaki y AYUDA, María Isabel (2006): “Una estimación del consumo de madera en España entre 1860 y 1935", Documento de Trabajo de la AEHE, DT-0603.

KREMERS, Jeroen M.; ERICSSON, Neil R., y DOLADO Juan J. (1992): “The power of cointegration tests", Oxford Bulletin of Economics and Statistics, 54, pp. 325358.

LATHAM, Bryan (1957): Timber, its development and distribution. A historical Survey, London, George G. Harrap.

LEGISLACIÓN DE ADUANAS (1922): Aranceles de importación y exportación para la Península e Islas Baleares vigentes desde 16 de febrero de 1922, Madrid, Góngora.

LIBECAP, Gary D., y JOHNSON, Ronald N. (1979): "Property Rights, NineteenthCentury Federal Timber Policy and the Conservation Movement", Journal of Economic History, 39, pp. 129-142.

LLEÓ, Antonio (1929): Las Realidades, las Posibilidades y las Necesidades Forestales de España, Madrid, Estudios Políticos, Sociales y Económicos.

MANUEL, Carlos M., y GIL, Luis (1998): “La transformación histórica del paisaje forestal en España", en MINISTERIO DE MEDIO AMBIENTE, Segundo inventario forestal nacional, 1986-1996, Madrid, Dirección General de Conservación de la Naturaleza, pp. 15-104.

M'HIRIT, Omar (1999): “El bosque mediterráneo: espacio ecológico, riqueza económica y bien social", Unasylva, 197, Depósitos de documentos de la FAO.

NADAL, Jordi (1987): "La industria fabril española en 1900. Una aproximación", en NADAL, Jordi; CARRERAS, Albert; y SUDRIÀ, Carles (comps.), La economía española en el siglo XX. Una perspectiva histórica, Barcelona, Ariel, pp. 23-61.

-(1990): "El desarrollo de la economía valenciana en la segunda mitad del XIX: ¿una vía exclusivamente agraria?", en NADAL, Jordi, y CARRERAS, Albert (eds.), Pautas regionales de la industrialización española (siglos XIX y XX), Barcelona, Ariel, pp. 296-314. 
NÁJERA, Fernando (1948): El abastecimiento del mercado nacional de maderas, Madrid, CSIC.

PARDOS, Eva (2001): “Una revisión de la polémica sobre las mediciones de la protección española en la Restauración", en SUDRIÀ, Carles, y TIRADO, Daniel (eds.), Peseta y protección. Comercio exterior, moneda y crecimiento económico en la España de la Restauración, Barcelona, Edicions UAB, pp. 71-91.

PAREJO, Francisco (2006): “Cambios en el negocio mundial corchero: un análisis a largo plazo de las exportaciones españolas (1849-2000)", Historia Agraria, 39, pp. 241-265.

PERLIN, John (1999): Historia de los bosques. El significado de la madera en el desarrollo de la civilización, Madrid, Gaia.

PRADOS, Leandro (1986): “Una serie anual del comercio exterior español (18211913)" Revista de Historia Económica, IV, 1, pp. 103-150.

-(1988): De imperio a nación. Crecimiento y atraso económico en España (1780-1930), Madrid, Alianza.

-(2004): El progreso económico de España, 1850-2000, Bilbao, Fundación BBVA.

SABATÉ, Marcela (1996): El proteccionismo legitimado. Política arancelaria española a comienzos de siglo, Madrid, Civitas.

SABATÉ, Marcela y PARDOS, Eva (2001): “Una cuestión a debatir. El nuevo perfil del proteccionismo español durante la Restauración", Revista de Historia Económica, XIX, 1, pp. 155-165.

SALA, Pere (1997): Sobre la compatibilitat entre bosc productor y bosc protector. La Catalunya forestal húmida entre la societat agraria y la societat industrial. 1850-1930, Tesis Doctoral, Universidad Autónoma de Barcelona.

SAWYER, Charles W., y SPRINKLE, Richard L. (1999): The demand for imports and exports in the world economy, Vermont, Ashgate Publishing Company.

SERRANO SANZ, José María (1987): El viraje proteccionista de la Restauración. La política comercial española, 1875-1895, Madrid, Siglo XXI.

SIEFERLE, Rolf P. (2001): "Qué es la historia ecológica", en GONZÁLEZ DE MOLINA, Manuel, y MARTÍNEZ ALIER, Joan (eds.), Naturaleza transformada, Barcelona, Icaria, pp. 31-54.

Statistical Abstract for the United Kingdom (varios números), London, Board of Trade.

TENA, Antonio (1999): “Un nuevo perfil del proteccionismo español durante la Restauración, 1875-1930", Revista de Historia Económica, XVIII, 3, pp. 579-621.

-(2001): "Réplica a Sabaté y Pardos", Revista de Historia Económica, XIX, 1, pp. 167172.

TIRADO, Daniel (1994): “La protección arancelaria en la Restauración. Nuevos indicadores", Revista de Economía Aplicada, 2, pp. 183-203.

-(1996): "Réplica a A. Tena", Revista de Economía Aplicada, 11, pp. 140-146. 
URIARTE, Rafael (1995): “La industrialización del bosque en la España interior: producción y cambio técnico en la industria resinera (1860-1914)", Revista de Historia Económica, XIII, 3, pp. 509-551.

WRIGLEY, Edward A. (1993): Cambio, continuidad y azar. Carácter de la revolución industrial inglesa, Barcelona, Crítica.

ZAPATA, Santiago (1998): Historia económica de la madera en España, desde mediados del siglo XIX a 1936. Un primer esbozo, Memoria de cátedra, Badajoz, Universidad de Extremadura.

-(2001): “La madera en España (c. 1850-c. 1950). Un primer esbozo", Revista de Historia Económica, XIX, 2, pp. 287-343. 
APÉNDICE

IMPORTACIONES, PROTECCIÓN Y PRECIOS DE LA MADERA

\begin{tabular}{|c|c|c|c|c|c|c|c|}
\hline & (1) & (2) & (3) & (4) & (5) & (6) & (7) \\
\hline 1880 & 28.759.308 & 3.784 .180 & 4,19 & 16,72 & 0,9 & 0,6 & 1,3 \\
\hline 1881 & 33.728 .573 & 3.879 .180 & 3,51 & 19,64 & 0,8 & 0,8 & 0,8 \\
\hline 1882 & 38.173 .186 & 4.583.378 & 3,36 & 20,07 & 0,9 & 0,8 & 0,8 \\
\hline 1883 & 44.613 .053 & 4.727 .627 & 3,44 & 21,97 & 0,8 & 0,7 & 1,0 \\
\hline 1884 & 41.070 .795 & 4.367 .470 & 3,15 & 21,44 & 0,8 & 0,5 & 0,8 \\
\hline 1885 & 33.360 .981 & 4.599 .503 & 4,57 & 19,89 & 0,8 & 0,5 & 0,9 \\
\hline 1886 & 39.562 .759 & 5.166 .998 & 3,20 & 19,24 & 0,7 & 0,6 & 0,8 \\
\hline 1887 & 35.485 .754 & 5.016 .879 & 3,03 & 20,34 & 0,7 & 0,6 & 0,8 \\
\hline 1888 & 30.851 .137 & 5.126 .498 & 2,96 & 21,31 & 0,7 & 0,6 & 0,8 \\
\hline 1889 & 43.295 .685 & 5.147 .714 & 3,00 & 24,15 & 0,8 & 0,7 & 0,8 \\
\hline 1890 & 44.016 .918 & 4.988 .852 & 2,93 & 23,33 & 0,8 & 0,7 & 0,8 \\
\hline 1891 & 43.003 .758 & 5.516 .181 & 3,03 & 20,15 & 0,7 & 0,6 & 0,8 \\
\hline 1892 & 39.729 .267 & 5.674 .306 & 5,98 & 22,57 & 0,7 & 0,5 & 0,9 \\
\hline 1893 & 33.328 .234 & 3.015 .848 & 8,60 & 32,45 & 0,7 & 0,5 & 0,9 \\
\hline 1894 & 37.443 .496 & 2.571 .261 & 5,95 & 43,72 & 0,7 & 0,5 & 0,8 \\
\hline 1895 & 37.396 .838 & 2.756 .913 & 5,35 & 39,73 & 0,7 & 0,4 & 0,8 \\
\hline 1896 & 29.967 .166 & 3.403 .482 & 6,55 & 33,08 & 0,7 & 0,4 & 1,0 \\
\hline 1897 & 31.417 .289 & 3.045 .184 & 7,01 & 32,83 & 0,8 & 0,4 & 0,9 \\
\hline 1898 & 22.963 .433 & 1.954 .466 & 8,82 & 35,15 & 0,8 & 0,5 & 0,8 \\
\hline 1899 & 37.333 .654 & 2.813 .672 & 7,27 & 41,91 & 0,8 & 0,7 & 0,8 \\
\hline 1900 & 48.806 .223 & 3.162 .995 & 6,00 & 39,73 & 0,9 & 1,1 & 1,0 \\
\hline 1901 & 49.196 .533 & 2.960 .509 & 6,15 & 40,13 & 0,8 & 0,6 & 1,0 \\
\hline 1902 & 38.159 .693 & 3.571 .389 & 6,24 & 32,64 & 0,8 & 0,7 & 1,0 \\
\hline 1903 & 46.061 .851 & 2.244 .180 & 6,15 & 40,66 & 0,9 & 0,9 & 1,0 \\
\hline 1904 & 45.363 .911 & 3.556 .727 & 8,62 & 31,27 & 0,8 & 0,9 & 1,0 \\
\hline 1905 & 33.040 .893 & 3.800 .767 & 8,63 & 26,16 & 0,8 & 0,9 & 0,9 \\
\hline 1906 & 37.392 .708 & 4.517 .397 & 7,28 & 17,00 & 0,9 & 0,9 & 1,0 \\
\hline 1907 & 44.685 .420 & 3.091 .357 & 7,86 & 13,98 & 1,0 & 0,9 & 1,0 \\
\hline 1908 & 44.792 .293 & 2.641 .637 & 7,65 & 19,75 & 0,9 & 0,6 & 1,0 \\
\hline 1909 & 46.483 .604 & 3.579 .535 & 7,45 & 15,18 & 0,9 & 0,7 & 1,0 \\
\hline 1910 & 47.410 .195 & 2.976 .602 & 6,84 & 15,71 & 1,0 & 1,0 & 1,0 \\
\hline 1911 & 55.499 .356 & 3.235 .786 & 6,54 & 18,54 & 1,0 & 0,9 & 1,0 \\
\hline
\end{tabular}


APÉNDICE (continuación)

IMPORTACIONES, PROTECCIÓN Y PRECIOS DE LA MADERA

\begin{tabular}{|c|c|c|c|c|c|c|c|}
\hline & (1) & (2) & (3) & (4) & (5) & (6) & (7) \\
\hline 1912 & 46.204 .732 & 3.834 .990 & 6,52 & 15,93 & 1,0 & 0,7 & 1,0 \\
\hline 1913 & 56.274 .403 & 3.651 .648 & 6,64 & 14,40 & 1,1 & 0,6 & 1,0 \\
\hline 1914 & 32.383 .186 & 2.644 .267 & 9,53 & 13,05 & 1,1 & 0,7 & 1,1 \\
\hline 1915 & 31.066 .783 & 2.366 .610 & 10,66 & 11,06 & 1,6 & 0,8 & 1,2 \\
\hline 1916 & 28.771 .089 & 2.832.077 & 12,92 & 10,60 & 2,7 & 0,9 & 1,5 \\
\hline 1917 & 14.337 .590 & 3.047 .806 & 12,41 & 10,17 & 3,7 & 1,3 & 1,9 \\
\hline 1918 & 10.263 .360 & 1.317 .672 & 3,56 & 5,63 & 4,9 & 2,4 & 2,0 \\
\hline 1919 & 11.989 .397 & 2.559 .419 & 4,72 & 7,86 & 3,9 & 2,0 & 2,2 \\
\hline 1920 & 16.860 .021 & 4.970 .959 & 7,72 & 20,51 & 4,2 & 3,3 & 2,2 \\
\hline 1921 & 12.500 .225 & 5.429 .524 & 11,08 & 18,90 & 1,7 & 1,6 & 2,3 \\
\hline 1922 & 38.025 .708 & 3.641 .277 & 23,78 & 21,23 & 1,8 & 1,8 & 2,2 \\
\hline 1923 & 46.792 .788 & 4.519 .872 & 29,13 & 28,91 & 1,6 & 1,8 & 2,3 \\
\hline 1924 & 50.437 .114 & 3.441 .910 & 31,86 & 39,61 & 1,4 & 1,5 & 2,3 \\
\hline 1925 & 66.291 .531 & 3.918 .857 & 34,05 & 29,57 & 1,2 & 1,5 & 2,4 \\
\hline 1926 & 63.199 .714 & 3.568 .208 & 31,28 & 32,57 & 1,2 & 1,4 & 2,0 \\
\hline 1927 & 68.821 .712 & 3.835 .826 & 23,66 & 26,31 & 1,6 & 1,0 & 1,9 \\
\hline 1928 & 79.224 .305 & 3.491 .759 & 26,01 & 28,26 & 1,6 & 0,8 & 1,9 \\
\hline 1929 & 86.172 .137 & 2.671 .405 & 30,43 & 35,55 & 1,6 & 0,8 & 2,0 \\
\hline 1930 & 71.321 .156 & 2.154 .732 & 36,08 & 44,64 & 1,5 & 0,7 & 2,0 \\
\hline 1931 & 42.411 .404 & 1.522 .318 & 43,48 & 49,68 & 1,2 & 0,6 & 2,1 \\
\hline 1932 & 44.246 .389 & 1.236 .793 & 47,87 & 37,43 & 1,1 & 0,9 & 2.0 \\
\hline 1933 & 38.815 .245 & 1.148 .898 & 39,82 & 34,34 & 1,1 & 0,8 & 2,0 \\
\hline 1934 & 39.859 .987 & 1.279 .949 & 36,43 & 37,88 & 1,2 & 0,7 & 2,0 \\
\hline 1935 & 41.990 .138 & 1.167 .421 & 36,85 & 38,32 & 1,1 & 0,7 & 2,0 \\
\hline
\end{tabular}

(1) y (2) Volúmenes de importaciones de madera no manufacturada y manufacturada, respectivamente. Cantidades multiplicadas por sus precios de importación del año 1910.

(3) y (4) Protección ad valorem corregida de la madera no manufacturada y manufacturada, respectivamente.

(5) y (6) Series de precios de importación británicos de la madera no manufacturada y manufacturada, respectivamente, base $1=1910$.

(7) Serie de precios interiores de la madera no manufacturada en España, base $1=1910$.

Fuentes: Para (1) y (2), Estadísticas del comercio exterior de España. Para (3) y (4), véase la tercera sección del artículo. Para (5) y (6), Statistical Abstract for the United Kingdom. Para (7), Zapata (1998) y Sala (1997). 